\title{
Allocating Capacity with Demand Competition: Fixed Factor Allocation*
}

\author{
Jianbin Li and Xueyuan Cai
}

School of Management, Huazhong University of Science and Technology, Wuhan, Hubei, 430074,China,e-mail: jbli@hust.edu.cn,xueyuancai@hust.edu.cn

\author{
Zhixin $\operatorname{Liu}^{\dagger}$ \\ College of Business, University of Michigan-Dearborn, Dearborn, MI 48126-2638, \\ e-mail:zhixin@umich.edu
}

\begin{abstract}
We consider a supply chain consisting of a supplier and two retailers. The supplier sells a single product to the retailers, who, in turn, retail the product to customers. The supplier has limited production capacity, and the retailers compete for the supplier's capacity and are duopolists engaged in Cournot competition for their customers. When the sum of the retailers' orders exceeds the supplier's capacity, the supplier allocates his capacity according to a preannounced allocation rule. We propose a new capacity allocation rule, fixed factor allocation, which incorporates the ideas of proportional and lexicographic allocations: it prioritizes retailers as in lexicographic allocation, but guarantees only a fixed proportion of the total available capacity to the prioritized retailer. We show that (1) the fixed factor allocation rule incorporates lexicographic and proportional allocations from the perspectives of the supplier and the supply chain; (2) under fixed factor allocation, the supply chain profit is not affected by the allocation factor when it is greater than a threshold; (3) the retailers share the supply chain profit with the supplier depending on the value of the allocation factor; and (4) the fixed factor allocation coordinates the supply chain when the market size is sufficiently large. We also compare fixed factor with proportional and lexicographic allocations, respectively. Furthermore, we demonstrate how the supplier can optimize his capacity level and wholesale price under fixed factor allocation. [Submitted: May 19, 2015. Revised: April 20, 2016. Accepted: May 10, 2016.]
\end{abstract}

Subject Areas: Allocation Rule, Capacity Allocation, Cournot Competition, Fixed Factor Allocation, Supply Chain, and Wholesale Pricing.

* The authors are sincerely grateful to the associate editor and two anonymous reviewers for their constructive comments, which substantially improved the quality of this work. The research was partly supported by the Program for New Century Excellent Talents in University (No. NCET-13-0228) and the Natural Science Foundation of China (Nos. 71571079, 71171088, 71131004).

${ }^{\dagger}$ Corresponding author. 


\section{INTRODUCTION}

In practice, a supplier with limited capacity often puts capacity on allocation, i.e., rationing capacity through quantity competition of retailers rather than through a pricing mechanism. Capacity allocation is a common occurrence in industries in which capacity expansion is costly and time-consuming and price is given exogenously (e.g., for steel and paper). A supplier can use his prior beliefs on his own and the retailers' needs to construct a capacity allocation mechanism for allocation of his capacity among retailers. Commonly used capacity allocation mechanisms contain allocation rules that allocate capacity based on retailers' order sizes, such as proportional allocation and lexicographic allocation. When the supplier's capacity is insufficient to fill all the orders received, proportional allocation allocates capacity in proportion to order size, and lexicographic allocation allocates capacity in the order of a predetermined priority sequence.

Lexicographic and proportional allocation mechanisms are widely used by companies in practice. Even though lexicographic allocation dominates proportional allocation in terms of increasing the profits for both the supplier and the supply chain when there exists downstream competition (Chen, Li, \& Zhang, 2013), lexicographic allocation treats retailers with identity discrimination and thus puts the supplier under risk of losing future business from some retailers. Specifically, retailers with low priority may turn to other suppliers for better wholesale price and order fulfillment. In addition, lexicographic allocation can easily drive retailers with low priority out of the market, and with fewer retailers retaining in the market, the supplier will suffer from reduced order size. Thus, these potential drawbacks motivate us to propose a new capacity allocation rule, namely, fixed factor allocation. Fixed factor allocation incorporates the principles underlying both proportional and lexicographic allocations: it prioritizes retailers as in lexicographic allocation, but guarantees only a fixed proportion of the total available capacity to the prioritized retailer, as in proportional allocation. The allocation corresponds with existing practice in pharmaceutical industry. For example, Zhong and Wu (2013) report that a manufacturer of Pien Tze Huang (traditional Chinese medicine) allocated restricted capacity to distributors based on fixed proportions. Due to the scarcity of the raw material, the short supply of Pien Tze Huang often occurred, in which cases the manufacturer guaranteed each distributor a fixed proportion of the total capacity, which resembles a fixed factor allocation.

We consider a supply chain consisting of a supplier and two retailers. The supplier sells a product to the two retailers, and then the retailers sell the product to customers in a common market. The supplier has limited capacity. If the total order size from the two retailers does not exceed the supplier's capacity, then each retailer receives what she orders; otherwise, the supplier allocates his capacity between the two retailers using a preannounced capacity allocation rule. We assume that the retail prices charged by the two retailers depend on the quantity of the product they each receive from the supplier, which is equal to the quantity they each sell in the market. Thus, the two retailers face order quantity competition, i.e., Cournot competition.

The decision sequence of the supplier and retailers is as follows. First, the supplier determines a wholesale price and announces his capacity level and a 
capacity allocation rule. Second, the two retailers submit their order quantities simultaneously. This constitutes a Stackelberg game, with the supplier as leader and the two retailers as followers. We investigate how the supplier optimizes his wholesale price to maximize his value as a leader and how the two retailers compete for limited capacity in their order quantity determination to maximize their values. We specifically study how the proposed fixed factor allocation rule affects the decisions of the supplier and the retailers. We also compare the fixed factor allocation with proportional and lexicographic allocations, respectively. We show that the fixed factor allocation rule incorporates both proportional and lexicographic allocations from the perspectives of the supplier and the whole supply chain, but not necessarily the retailers. Under fixed factor allocation, we demonstrate that the supply chain profit is not affected by the allocation factor when it is greater than a threshold, and the retailers can share the supply chain profit with the supplier by negotiating the allocation factor value.

Capacity allocation mechanisms have been employed widely in industries, including automobiles, pharmaceuticals, and toys (e.g., Hwang \& Valeriano, 1992; Blumenstein, 1996). For the properties of a large variety of capacity allocation rules, we refer to the survey paper by Hall and Liu (2010). Next, we review the studies on proportional and lexicographic allocation rules, which are also examined in our work. Lee, Padmanabhan, and Whang (1997) recognize that proportional allocation creates incentive for retailers to raise their orders above their desired allocations in multiechelon supply chains. Cachon and Lariviere (1999a) demonstrate that proportional allocation with fixed price can lead retailers to order more than they desire to receive a favorable allocation, even when they directly order from the supplier. These authors also show that lexicographic allocation is truth-inducing in that it provides no incentive for retailers to order more than they desire. However, under proportional allocation, both the supplier and the whole supply chain can earn higher profits. These authors further demonstrate that a truth-inducing allocation with fixed price cannot maximize retailers' total profit. Cachon and Lariviere (1999b) more specifically compare proportional and lexicographic allocations. These authors show that whether order inflation incentivized by proportional allocation helps or harms a supply chain depends on how profits are distributed within the supply chain. In general, encouraging order inflation increases supplier's profit but decreases retailers' profits.

The following three papers on capacity allocation are closely related to this article in that they consider demand competition among retailers. Liu (2012) studies how different capacity allocation rules affect profits of different supply chain members, where retail prices linearly depend on the total sales volume of two competing retailers. An interesting finding is that the supplier can sell more with less capacity, and retailers may earn more when the supplier has less capacity, due to the demand competition between the two retailers. Cho and Tang (2014) develop an important extension of Liu (2012)'s work by solving the case with multiple retailers. These authors specifically investigate the gaming effect caused by uniform allocation. They also propose a new allocation, namely, competitive allocation, which can eliminate the gaming effect with demand competition among retailers. Chen et al. (2013) consider capacity allocation in a supply chain consisting of a supplier and multiple retailers with the same market power in demand competition. 
These authors consider the supplier's wholesale pricing decision when using proportional and lexicographic allocations. They show that lexicographic allocation can generate higher profits for both the supplier and the supply chain.

Models for capacity allocation in different settings either illustrate the computational difficulties of achieving a desirable allocation (e.g., Clark \& Scarf, 1960), or show that a desirable allocation can be obtained (e.g., Eppen \& Schrage, 1981; Federgruen \& Zipkin, 1984; Jonsson \& Silver, 1987; Schwarz, 1989; Chen \& Zheng, 1994; Kumar, Schwarz, \& Ward, 1995). Note that the definition of desirable allocation varies from model to model. Several author groups (e.g., Topkis, 1969; Ha, 1997; Deshpande \& Schwarz, 2002; Deshpande, Cohen, \& Donohue, 2003) examine allocation of inventory with sequentially arriving customers of different priority classes in a centralized setting. Cachon and Lariviere (1999c) investigate a turn-and-earn allocation mechanism over two periods, where capacity allocation in the second period is based on sales volume in the first period.

The remainder of this article proceeds as follows. In section "Model Description," we define the model and introduce the fixed factor allocation rule. Retailers' best response decisions in Nash equilibrium are derived in section "Retailers' Decisions." In section "Supplier's Decision," we characterize the supplier's optimal decision. In section "Impacts of Fixed Factor $\alpha$," we investigate how allocation factor affects profits of different supply chain members. In section "Comparison with Centralized Supply Chain," we compare the supply chain profit under fixed factor allocation with the profit of a centralized supply chain. We then compare fixed factor allocation with proportional and lexicographic allocations, respectively, in section "Comparison with Other Allocations," and study the optimal capacity choice of the supplier in section "Optimal Capacity Choice." Finally, concluding remarks and suggestions for future research are set forth in the last section. All proofs are in the Appendix.

\section{MODEL DESCRIPTION}

We consider a one-period setting in which a single supplier sells one product to two retailers, who, in turn, retail the product to customers in a common market. The quantity ordered by a retailer may not be the same as what she finally receives, which depends on how the supplier allocates his capacity when the total order quantity by the two retailers exceeds his capacity. We assume that order quantity by each retailer cannot exceed the supplier's capacity and the retail price is market size minus the total quantity that the supplier allocates to the two retailers. Specifically, for each retailer $i \in\{1,2\}$, given the other retailer's allocated quantity, the market price is decreasing in her own allocated quantity, and consequently her profit function is concave with her allocated quantity.

Prior to the decision period, we have the following assumptions. First, the supplier has a fixed capacity size. Second, the supplier preannounces publicly the allocation rule he will use to allocate his capacity. While both the capacity level and allocation rule are taken as given in our model, essentially they could be determined by the supplier, who wants to maximize his own profit. We investigate the supplier's decision on capacity level and allocation rule. 
During the decision period, events occur in the following sequence. First, the supplier determines the wholesale price. Second, the two retailers determine their order quantities and simultaneously submit them to the supplier. Third, the supplier fills orders according to the preannounced allocation rule. Fourth, retailers sell the products in a common market and realize their profits.

Note that retailers submit orders independently, and orders are the only communication between the retailers and the supplier. No retailer can credibly announce her information to other players, including the supplier and the other retailer, and no side contract between the supplier and any retailer is allowed. In short, a retailer can influence her allotment and the other retailer's allotment only through her order. The supplier charges a wholesale price that is determined after he chooses an allocation rule, and a retailer must accept the price and pay for any allocation up to her full order. The supplier cannot deliver to a retailer more than she has ordered.

We use the following notation throughout the article:

$K$ : the supplier's capacity,

$w$ : the wholesale price,

$M$ : the market size,

$m_{i}$ : retailers $i$ 's order quantity, $i=1,2$,

$m$ : order vector $m=\left(m_{1}, m_{2}\right)$,

$g(m)$ : allocation rule defined by function $g$ for order vector $m$,

$g_{i}(m)$ : retailer $i$ 's allocated quantity under allocation function $g$ for order vector $m, i=1,2$,

$c$ : unit production cost.

Recall that each retailer's order quantity is no more than the supplier's capacity, i.e., $m_{i} \leq K, i=1,2$. An allocation rule $g(m) \equiv g\left(m_{1}, m_{2}\right)$ is a function of the retailers' order vector $m=\left(m_{1}, m_{2}\right)$, and defines an allocation vector $\left(g_{1}(m), g_{2}(m)\right)$. Note that for an allocation vector to be feasible, we require that $\sum_{i=1}^{2} g_{i}(m) \leq K$. Also, the supplier can never allocate to a retailer more than her order quantity, i.e., $g_{i}(m) \leq m_{i}, i=1,2$.

Next, we introduce three allocation rules: proportional, lexicographic, and fixed factor allocations. Proportional allocation allocates capacity in proportion to order size if capacity is insufficient to fill all orders. Specifically, we have

$$
\begin{aligned}
& g_{1}\left(m_{1}, m_{2}\right)= \begin{cases}m_{1} & \text { if } m_{1}+m_{2} \leq K, \\
\frac{m_{1}}{m_{1}+m_{2}} K & \text { if } m_{1}+m_{2}>K,\end{cases} \\
& g_{2}\left(m_{1}, m_{2}\right)= \begin{cases}m_{2} & \text { if } m_{1}+m_{2} \leq K, \\
\frac{m_{2}}{m_{1}+m_{2}} K & \text { if } m_{1}+m_{2}>K .\end{cases}
\end{aligned}
$$

Lexicographic allocation prioritizes retailers and always tries to fill the order of the retailer with the highest priority. Without loss of generality, we assume that 
retailer 1 is granted a priority higher than retailer 2 . We have

$$
\begin{gathered}
g_{1}\left(m_{1}, m_{2}\right)=m_{1} \wedge K, \\
g_{2}\left(m_{1}, m_{2}\right)=m_{2} \wedge\left(K-m_{1}\right) .
\end{gathered}
$$

Now, we define our fixed factor allocation rule. The fixed factor allocation incorporates the principles of proportional and lexicographic allocations: it prioritizes retailers as in lexicographic allocation, but guarantees only a fixed proportion of the total available capacity to the prioritized retailer, as in proportional allocation. We denote $0 \leq \alpha \leq 1$ as the proportion of total capacity reserved for the retailer with higher priority. Without loss of generality, we assume that retailer 1 is granted a priority higher than retailer 2 . For any order vector $m=\left(m_{1}, m_{2}\right)$, the fixed factor allocation rule is given by

$$
\begin{gathered}
g_{1}\left(m_{1}, m_{2}\right)=\left\{\begin{array}{lll}
m_{1} & \text { if } & m_{1}+m_{2} \leq K \text { or } m_{1}+m_{2}>K, m_{1} \leq \alpha K \\
\alpha K & \text { if } & m_{1}>\alpha K, m_{2}>(1-\alpha) K, \\
K-m_{2} & \text { if } & m_{1}+m_{2}>K, m_{2} \leq(1-\alpha) K,
\end{array}\right. \\
g_{2}\left(m_{1}, m_{2}\right)=\left\{\begin{array}{lll}
m_{2} & \text { if } & m_{1}+m_{2} \leq K \text { or } m_{1}+m_{2}>K, m_{2} \leq(1-\alpha) K, \\
(1-\alpha) K & \text { if } & m_{1}>\alpha K, m_{2}>(1-\alpha) K, \\
K-m_{1} & \text { if } & m_{1}+m_{2}>K, m_{1} \leq \alpha K .
\end{array}\right.
\end{gathered}
$$

When capacity is insufficient, the fixed factor allocation rule guarantees an allocation of a certain proportion of capacity to each retailer, i.e., $\alpha K$ to retailer with high priority, $(1-\alpha) K$ to retailer 2 with low priority. If retailer $i$ orders less than its guaranteed allocation, she will receive the quantity she orders; otherwise, she will receive at least the guaranteed quantity.

Note that we consider two symmetric retailers. We regard retailer 1 as prioritized with higher priority in capacity allocation, and hence assume that $\alpha \in[1 / 2,1]$. Fixed factor allocation degenerates to lexicographic allocation when $\alpha=1$, and to uniform allocation (see, e.g., Hall \& Liu, 2010) when $\alpha=1 / 2$.

For allocation rule $g(\cdot)$, the market price of the product is expressed as

$$
M-g_{1}\left(m_{1}, m_{2}\right)-g_{2}\left(m_{1}, m_{2}\right),
$$

where $M$ represents market size. The supplier's problem is to choose a wholesale price $w$ to maximize his profit,

$$
\Pi_{s}(\alpha)=\max _{w}\left\{w \cdot\left(g_{1}\left(m_{1}, m_{2}\right)+g_{2}\left(m_{1}, m_{2}\right)\right)\right\} ;
$$

while retailers simultaneously choose their order quantities $\left(m_{1}, m_{2}\right)$ to maximize their individual profit as follows:

$$
\begin{aligned}
& \Pi_{1}\left(w, m_{2}\right)=\max _{m_{1}}\left(M-g_{1}\left(m_{1}, m_{2}\right)-g_{2}\left(m_{1}, m_{2}\right)-w\right) g_{1}\left(m_{1}, m_{2}\right), \\
& \Pi_{2}\left(w, m_{1}\right)=\max _{m_{2}}\left(M-g_{1}\left(m_{1}, m_{2}\right)-g_{2}\left(m_{1}, m_{2}\right)-w\right) g_{2}\left(m_{1}, m_{2}\right) .
\end{aligned}
$$


Here, the supplier and two retailers constitute a Stackelberg game with the supplier as leader and the two retailers as followers. Meanwhile, the two retailers play a Cournot game in a common retail market.

\section{RETAILERS' DECISIONS}

In this section, we analyze the best response function of each retailer given wholesale price and the other retailer's order quantity, and then characterize the two retailers' equilibrium order quantities for a given wholesale price, under fixed factor allocation by the supplier. Because the fixed factor allocation rule is not symmetric for the two retailers, retailer decisions will differ. Thus, we investigate the decisions of the two retailers separately. In the remainder of this section, we analyze the decisions of retailer 1 (with higher priority in capacity allocation), retailer 2 (with lower priority in capacity allocation), and the two retailers in equilibrium, respectively.

\section{Retailer 1's Best Response Function}

From Equation (8), it is difficult to determine the concavity of retailer 1's profit function. Thus, we analyze the profit function (8) by dividing the problem into two scenarios: Scenario 1: $m_{1}+m_{2} \leq K$ (i.e., retailers' total order quantity is no greater than the supplier's capacity); and Scenario $2: m_{1}+m_{2}>K$ (i.e., retailers' total order quantity is greater than the supplier's capacity).

We can rewrite retailer 1's profit function as follows under fixed factor allocation:

$$
\begin{aligned}
\Pi_{1}\left(w, m_{2}\right) & =\max _{m_{1}}\left(M-g_{1}\left(m_{1}, m_{2}\right)-g_{2}\left(m_{1}, m_{2}\right)-w\right) g_{1}\left(m_{1}, m_{2}\right) \\
& =\max \left\{\max _{m_{1} \leq K-m_{2}} G_{11}\left(m_{1}, m_{2}\right), \max _{m_{1} \in\left(K-m_{2}, K\right]} G_{12}\left(m_{1}, m_{2}\right)\right\},
\end{aligned}
$$

where

$$
\begin{aligned}
& G_{11}\left(m_{1}, m_{2}\right)=\left(M-m_{1}-m_{2}-w\right) m_{1}, \\
& G_{12}\left(m_{1}, m_{2}\right)=\left\{\begin{array}{l}
(M-K-w)\left(K-m_{2}\right) \quad m_{2} \leq(1-\alpha) K \\
(M-K-w)\left(m_{1} \wedge \alpha K\right) m_{2}>(1-\alpha) K,
\end{array}\right.
\end{aligned}
$$

where $G_{11}\left(m_{1}, m_{2}\right)$ represents retailer 1's profit in scenario 1, in which the total order quantity of two retailers is no more than the supplier's capacity $K$, and $G_{12}\left(m_{1}, m_{2}\right)$ denotes retailer 1's profit in scenario 2, in which the two retailers' total order quantity is greater than $K$. Note that in scenario 1 , each retailer obtains her desired order quantity, while in scenario 2, each retailer is allocated a quantity as specified by fixed factor allocation.

It is straightforward to determine the optimal solution of $m_{1}$ for profit function $G_{11}\left(m_{1}, m_{2}\right)$ in scenario 1 . If retailer 2 orders a small quantity (i.e., $m_{2} \leq 2 K-$ $(M-w)$ and $\left.m_{2} \leq M-w\right)$, then retailer 1 will order her desired quantity $(M-$ $\left.m_{2}-w\right) / 2$ and gains an optimal profit $\left(M-w-m_{2}\right)^{2} / 4$; if retailer 2 orders a very large quantity (i.e., $\left.m_{2}>M-w\right)$, then retailer 1 will order nothing and 
gains no profit. In addition, if retailer 2 orders a moderately large quantity (i.e., $\left.m_{2}>2 K-(M-w)\right)$, then retailer 1 will order just the surplus capacity $\left(K-m_{2}\right)$ to gain an optimal profit of $(M-K-w)\left(K-m_{2}\right)$.

Next, we maximize profit function $G_{12}$ in scenario 2, as in the following lemma.

Lemma 1: Let $m_{12}^{*}$ and $G_{12}^{*}$ be the optimal order quantity and profit of the function $G_{12}$ for given $w$ and $m_{2}$, respectively.

(i) Suppose $m_{2} \leq(1-\alpha) K$ :

$m_{12}^{*} \in\left(K-m_{2}, K\right]$ and $G_{12}^{*}=(M-K-w)\left(K-m_{2}\right)$.

(ii) Suppose $m_{2} \in((1-\alpha) K, K]$ :

if $w<M-K$, then $m_{12}^{*} \in[\alpha K, K]$ and $G_{12}^{*}=(M-K-w) \alpha K$;

if $w \geq M-K$, then $m_{12}^{*}=\left(K-m_{2}\right)^{+}$and $G_{12}^{*}=(M-K-w)(K-$ $\left.m_{2}\right)^{+}$.

Lemma 1 illustrates retailer 1's best response function when the total order quantity is greater than the supplier's capacity $K$. Comparing results in Lemma 1 with those obtained under scenario 1, we can locate retailer 1's global best response function given retailer 2's order quantity in Theorem 1, as follows. Note that there are other ways to present the results of Theorem 1 . We develop Theorem 1 in terms of the wholesale price. For simplicity, let $\hat{\alpha}=M-w-2 \sqrt{(M-w-K) \alpha K}$. Note that $\hat{\alpha} \geq(1-\alpha) K$.

Theorem 1: Let $m_{1}\left(m_{2}\right)$ be retailer 1's best response function; i.e., if retailer 2 orders $m_{2}$, then it is optimal for retailer 1 to order $m_{1}\left(m_{2}\right)$.

(i) Suppose $w \in(M-K, M]$ :

if $m_{2} \in[0, M-w)$, then $m_{1}\left(m_{2}\right)=\frac{M-w-m_{2}}{2}$ and $\Pi_{1}\left(w, m_{2}\right)=$ $\frac{\left(M-w-m_{2}\right)^{2}}{4}$;

if $m_{2} \in[M-w, K]$, then $m_{1}\left(m_{2}\right)=0$ and $\Pi_{1}\left(w, m_{2}\right)=0$.

(ii) Suppose $w \in(M-(1+\alpha) K, M-K]$ :

if $m_{2} \in[0, \hat{\alpha}]$, then $m_{1}\left(m_{2}\right)=\frac{M-w-m_{2}}{2}$ and $\Pi_{1}\left(w, m_{2}\right)=\frac{\left(M-w-m_{2}\right)^{2}}{4}$;

if $m_{2} \in(\hat{\alpha}, K]$, then $m_{1}\left(m_{2}\right) \in[\alpha K, K]$ and $\Pi_{1}\left(w, m_{2}\right)=(M-K-$ $w) \alpha K$.

(iii) Suppose $w \in(M-2 K, M-(1+\alpha) K]$ :

if $m_{2} \in[0,2 K-(M-w))$, then $m_{1}\left(m_{2}\right)=\frac{M-w-m_{2}}{2}$ and $\Pi_{1}\left(w, m_{2}\right)=$ $\frac{\left(M-w-m_{2}\right)^{2}}{4}$;

if $m_{2} \in[2 K-(M-w),(1-\alpha) K)$, then $m_{1}\left(m_{2}\right) \in\left[K-m_{2}, K\right]$ and $\Pi_{1}\left(w, m_{2}\right)=(M-w-K)\left(K-m_{2}\right)$;

if $m_{2} \in[(1-\alpha) K, K]$, then $m_{1}\left(m_{2}\right) \in[\alpha K, K]$ and $\Pi_{1}\left(w, m_{2}\right)=$ $(M-w-K) \alpha K$.

(iv) Suppose $w \leq M-2 K$ :

if $m_{2} \in[0,(1-\alpha) K)$, then $m_{1}\left(m_{2}\right) \in\left[K-m_{2}, K\right]$ and $\Pi_{1}\left(w, m_{2}\right)=$ $(M-w-K)\left(K-m_{2}\right)$;

if $m_{2} \in[(1-\alpha) K, K]$, then $m_{1}\left(m_{2}\right) \in[\alpha K, K]$ and $\Pi_{1}\left(w, m_{2}\right)=$ $(M-w-K) \alpha K$. 
The intuition behind Theorem 1 is fairly easy to see. First, it is evident that if the wholesale price is high (i.e., $w>M-2 K$ ) and retailer 2 orders little, then retailer 1 will not order much either (just $\left(M-w-m_{2}\right) / 2$ ), and the capacity constraint does not affect her order decision. In this case, the best response function is the same as if there does not exist capacity constraint. This explains the first element in (i)-(iii), above. Also, if retailer 2 orders large $\left(m_{2} \geq M-w\right)$, then retailer 1 will order nothing because she cannot earn any positive profit, which explains the second part of (i). Second, if the capacity limit is exceeded by total order size with retailer 2's order quantity no less than $(1-\alpha) K$, then retailer 1 will order no less than $\alpha K$. This is because if total order quantity exceeds capacity, then the total supply to the market will be $K$, which means that the margin profit for each retailer is fixed at $M-K-w$, and consequently retailer 1's profit is increasing in her allocation, and thus she will order $[\alpha K, K]$ to maximize her allocation at $\alpha K$. This explains the last element in (ii)-(iv), above. Third, when $w$ is smaller and retailer 2 does not order much, retailer 1 will order no less than the surplus capacity $K-m_{2}$, as in the second part of (iii) and first part of (iv), above.

\section{Retailer 2's Best Response Function}

Because of the asymmetry of retailers caused by higher priority of retailer 1 in fixed factor allocation, we need to analyze retailer 2's best response function to derive the Nash equilibrium ordering quantities of the two retailers. We proceed to analyze retailer 2's best response function given the supplier's wholesale price and retailer 1's order quantity. Retailer 2 will choose her order quantity to maximize her profit function, defined by Equation (9), which we rewrite as follows:

$$
\begin{aligned}
\Pi_{2}\left(w, m_{1}\right) & =\max _{m_{2} \leq K}\left(M-g_{1}\left(m_{1}, m_{2}\right)-g_{2}\left(m_{1}, m_{2}\right)-w\right) g_{2}\left(m_{1}, m_{2}\right) \\
& =\max \left\{\max _{m_{2} \leq K-m_{1}} G_{21}\left(m_{1}, m_{2}\right), \max _{m_{2} \in\left(K-m_{1}, K\right]} G_{22}\left(m_{1}, m_{2}\right)\right\},
\end{aligned}
$$

where

$$
\begin{aligned}
& G_{21}\left(m_{1}, m_{2}\right)=\left(M-m_{1}-m_{2}-w\right) m_{2}, \\
& G_{22}\left(m_{1}, m_{2}\right)= \begin{cases}(M-K-w)\left(K-m_{1}\right) & m_{1} \leq \alpha K \\
(M-K-w)\left(m_{2} \wedge(1-\alpha) K\right) & m_{1}>\alpha K .\end{cases}
\end{aligned}
$$

Note that $G_{21}$ is symmetric with $G_{11}$, and we can maximize $G_{21}$ in a similar way as we maximize $G_{11}$, as in section "Retailer 1's Best Response Function," and hence we omit the details. Next, we maximize $G_{22}\left(m_{1}, m_{2}\right)$.

Lemma 2: Let $m_{22}^{*}$ and $G_{22}^{*}$ be the optimal order quantity and profit of the function $G_{22}$ for given $w$ and $m_{1}$, respectively.

(i) Suppose $m_{1} \leq \alpha K$ :

$m_{22}^{*} \in\left(K-m_{1}, K\right]$ and $G_{22}^{*}=(M-K-w)\left(K-m_{1}\right)$.

(ii) Suppose $m_{1} \in(\alpha K, K]$ :

if $w<M-K$, then $m_{22}^{*} \in[(1-\alpha) K, K]$ and $G_{22}^{*}=(M-K-$ $w)(1-\alpha) K$ 
if $w \geq M-K$, then $m_{22}^{*}=\left(K-m_{1}\right)^{+}$and $G_{22}^{*}=(M-K-w)(K-$ $\left.m_{1}\right)^{+}$.

In view of Lemma 2, we next characterize retailer 2's best response function for a given wholesale price and retailer 1's order quantity, as the wholesale price changes. Let

$$
\beta=M-w-2 \sqrt{(M-K-w)(1-\alpha) K} .
$$

Note that $\beta>\alpha K$.

Theorem 2: Let $m_{2}\left(m_{1}\right)$ be retailer 2's best response function, i.e., if retailer 1 orders $m_{1}$, then it is optimal for retailer 2 to order $m_{2}\left(m_{1}\right)$.

(i) Suppose $w \in(M-K, M]$ :

if $m_{1} \in[0, M-w)$, then $m_{2}\left(m_{1}\right)=\frac{M-w-m_{1}}{2}$ and $\Pi_{2}\left(w, m_{1}\right)=$ $\frac{\left(M-w-m_{1}\right)^{2}}{4}$;

if $m_{1} \in[M-w, K]$, then $m_{2}\left(m_{1}\right)=0$ and $\Pi_{2}\left(w, m_{1}\right)=0$.

(ii) Suppose $w \in(M-(2-\alpha) K, M-K]$ :

if $m_{1} \in[0, \beta]$, then $m_{2}\left(m_{1}\right)=\frac{M-w-m_{1}}{2}$ and $\Pi_{2}\left(w, m_{1}\right)=\frac{\left(M-w-m_{1}\right)^{2}}{4}$;

if $m_{1} \in(\beta, K]$, then $m_{2}\left(m_{1}\right) \in[(1-\alpha) K, K]$ and $\Pi_{2}\left(w, m_{1}\right)=(M-$ $K-w)(1-\alpha) K$.

(iii) Suppose $w \in(M-2 K, M-(2-\alpha) K]$ :

if $m_{1} \in[0,2 K-(M-w))$, then $m_{2}\left(m_{1}\right)=\frac{M-w-m_{1}}{2}$ and $\Pi_{2}\left(w, m_{2}\right)=$ $\frac{\left(M-w-m_{1}\right)^{2}}{4}$;

if $m_{1} \in[2 K-(M-w), \alpha K)$, then $m_{2}\left(m_{1}\right) \in\left[K-m_{1}, K\right] \quad$ and $\Pi_{2}\left(w, m_{1}\right)=(M-w-K)\left(K-m_{1}\right)$;

if $m_{1} \in[\alpha K, K]$, then $m_{2}\left(m_{1}\right) \in[(1-\alpha) K, K]$ and $\Pi_{2}\left(w, m_{1}\right)=$ $(M-w-K)(1-\alpha) K$.

(iv) Suppose $w \leq M-2 K$ :

if $m_{1} \in[0, \alpha K)$, then $m_{2}\left(m_{1}\right) \in\left[K-m_{1}, K\right]$ and $\Pi_{2}\left(w, m_{1}\right)=(M-$ $w-K)\left(K-m_{1}\right)$;

if $m_{1} \in[\alpha K, K]$, then $m_{2}\left(m_{1}\right) \in[(1-\alpha) K, K]$ and $\Pi_{2}\left(w, m_{1}\right)=$ $(M-w-K)(1-\alpha) K$.

We explain Theorem 2 as follows. First, it is evident that if the wholesale price is high (i.e., $w>M-2 K$ ) and retailer 1 orders little, then retailer 2 will order her desired quantity $\left(M-w-m_{1}\right) / 2$, because the capacity constraint is not binding. In this case, the best response function is the same as if there were no capacity limit; each retailer will order her desired quantity. This explains the first part in (i)-(iii), above. Also, it is intuitive that if retailer 1 orders more $\left(m_{1} \geq M-w\right)$, then retailer 2 will order nothing due to negative marginal profit, as reflected by the second part of (i), above. Second, if the wholesale price belongs to $[M-(2-\alpha) K, M-K]$, then retailer 2 will order no less than the guaranteed capacity $(1-\alpha) K$ when she assumes that retailer 1 's order quantity is more than $M-w-2 \sqrt{(M-K-w)(1-\alpha) K}$, as in the second part of (ii). Third, if the wholesale price is small, i.e., the marginal profit for the two retailers is large, then retailer 2 will order to receive the maximum possible allocation, ordering a quantity 
in $\left(K-m_{1}, K\right]$ assuming that retailer 1 's order quantity is small, and ordering a quantity in $[(1-\alpha) K, K]$ assuming that retailer 1 's order is sufficiently large. The results are reflected in the last two parts of (iii) and the whole of (iv), above.

\section{Nash Equilibrium}

With Theorems 1 and 2, we now characterize the two retailers' order quantities, which are in Nash equilibrium. Let $w^{c}(\alpha)=M-\gamma(\alpha)$, where $\gamma(\alpha)=$ $\frac{9 \alpha K-3 \sqrt{(9 \alpha-4) \alpha} K}{2}$. As will be seen, there are two scenarios based on the order between $w^{c}(\alpha)$ and $M-(2-\alpha) K$. What is more, $w^{c}(\alpha)$ is a critical value of wholesale price that affects the retailers' ordering decisions in equilibrium.

Theorem 3: Denote $\left(m_{1}^{*}, m_{2}^{*}\right)$ as an order pair in Nash equilibrium, we have:

(i) Suppose $1 / 2 \leq \alpha \leq 4 / 5$ (i.e., $w^{c}(\alpha) \geq M-(2-\alpha) K$ )

(i.a) if $w \leq M-(1+\alpha) K$, then there exist multiple Nash equilibria $\left.m_{1}^{*} \times m_{2}^{*} \in[\alpha K, K] \times[(1-\alpha) K), K\right]$

(i.b) if $w \in(M-(1+\alpha) K, M-(2-\alpha) K]$, then there exist multiple Nash equilibria $m_{1}^{*} \times m_{2}^{*} \in[\alpha K, K] \times[\hat{\alpha}, K]$

(i.c) if $w \in\left(M-(2-\alpha) K, w^{c}(\alpha)\right)$, then there exist multiple Nash equilibria $m_{1}^{*} \times m_{2}^{*} \in[\beta, K] \times[\hat{\alpha}, K]$

(i.d) if $w \in\left[w^{c}(\alpha), M-K\right]$, then there exist multiple Nash equilibria $\left(m_{1}^{*}, m_{2}^{*}\right)=\left(\frac{M-w}{3}, \frac{M-w}{3}\right)$ and $m_{1}^{*} \times m_{2}^{*} \in[\beta, K] \times[\hat{\alpha}, K]$. In the later set of equilibria, retailers 1 and 2 are allocated $\alpha K$ and $(1-\alpha) K$, respectively. Furthermore, the former equilibrium $\left(\frac{M-w}{3}, \frac{M-w}{3}\right)$ dominates any equilibrium in the later set of equilibria in that it brings higher profit to each retailer;

(i.e) if $w>M-K$, then there exists a unique Nash equilibrium $\left(m_{1}^{*}, m_{2}^{*}\right)=\left(\frac{M-w}{3}, \frac{M-w}{3}\right)$ and the total order quantity of the two retailers is less than the supplier's capacity level $K$.

(ii) Suppose $4 / 5<\alpha \leq 1$ (i.e., $\left.w^{c}(\alpha)<M-(2-\alpha) K\right)$

(ii.a) if $w \leq M-(1+\alpha) K$, then there exist multiple Nash equilibria $\left.m_{1}^{*} \times m_{2}^{*} \in[\alpha K, K] \times[(1-\alpha) K), K\right]$

(ii.b) if $w \in\left(M-(1+\alpha) K, w^{c}(\alpha)\right)$, then there exist multiple Nash equilibria $m_{1}^{*} \times m_{2}^{*} \in[\alpha K, K] \times[\hat{\alpha}, K]$

(ii.c) if $w \in\left[w^{c}(\alpha), M-(2-\alpha) K\right]$, then there exist multiple Nash equilibria $\left(m_{1}^{*}, m_{2}^{*}\right)=\left(\frac{M-w}{3}, \frac{M-w}{3}\right)$ and $m_{1}^{*} \times m_{2}^{*} \in[\alpha K, K] \times[\hat{\alpha}, K]$. In the later set of equilibria, retailers 1 and 2 are allocated $\alpha K$ and $(1-\alpha) K$, respectively. Furthermore, the former equilibrium $\left(\frac{M-w}{3}, \frac{M-w}{3}\right)$ dominates any equilibrium in the later set of equilibria in that it brings higher profit to each retailer;

(ii.d) if $w \in(M-(2-\alpha) K, M-K]$, then there exist multiple Nash equilibria $\left(m_{1}^{*}, m_{2}^{*}\right)=\left(\frac{M-w}{3}, \frac{M-w}{3}\right)$ and $m_{1}^{*} \times m_{2}^{*} \in[\beta, K] \times$ $[\hat{\alpha}, K]$. In the later set of equilibria, retailers 1 and 2 are allocated $\alpha K$ and $(1-\alpha) K$, respectively. Furthermore, the former equilibrium $\left(\frac{M-w}{3}, \frac{M-w}{3}\right)$ dominates any equilibrium in the later set of equilibria in that it brings higher profit to each retailer; 
(ii.e) if $w>M-K$, then there exists a unique Nash equilibrium $\left(m_{1}^{*}, m_{2}^{*}\right)=\left(\frac{M-w}{3}, \frac{M-w}{3}\right)$ and the total order quantity of the two retailers is less than the supplier's capacity level $K$.

Note that if $w<w^{c}(\alpha)$, then the profit of each retailer and the supplier is the same at any equilibrium order pair in $(\alpha K,(1-\alpha) K)$.

Theorem 3 is in terms of the wholesale price $w$ and conveniently describes the retailers' total order quantity as a function of the wholesale price. For $w<w^{c}(\alpha)$, total order quantity is $K$. That is, the supplier's capacity will be used up when the wholesale price is low. While the wholesale price is slightly higher $\left(w \geq w^{c}(\alpha)\right)$, the total quantity ordered is $2(M-w) / 3$, which achieves its maximum value of $2 \gamma(\alpha) / 3$ when $w=w^{c}(\alpha)$. Note that the retailers' total order quantity is a nonincreasing function of the wholesale price, as expected. What is interesting is that as the wholesale price crosses the critical level $w^{c}(\alpha)$, total order quantity drops from $K$ to a value strictly less than $K$. This discontinuity is due to the concurrent presence of the capacity limit by the supplier and the competition between the two retailers.

As Theorem 3 shows, there exist multiple Nash equilibria in the two retailers' ordering. For cases (i.a),(i.b), and (i.c) of the theorem, in all equilibria, retailer 1 orders no less than $\alpha K$, if $w \leq M-(2-\alpha) K$, then retailer 1 orders more than $\alpha K$; while if $w \in\left(M-(2-\alpha) K, w^{c}(\alpha)\right)$, then retailer 1 orders no less than $\beta$ to reach an equilibrium, and in both cases, it receives the maximum possible allocation from the supplier, as specified by the allocation factor $\alpha$. Accordingly, if $w \leq M-(1+\alpha) K$, then retailer 2 orders more than $(1-\alpha) K$; while if $w \in$ $\left(M-(1+\alpha) K, w^{c}(\alpha)\right)$, then retailer 2 orders at least $\hat{\alpha}$ to reach an equilibrium; and both cases bring retailer 2 an allocation of $(1-\alpha) K$. Note that all these equilibria lead to the same profits to all the supply chain members as when the two retailers order $(\alpha K,(1-\alpha) K)$. For case (i.d) of Theorem 3, we proceed by adopting the equilibrium $\left(\frac{M-w}{3}, \frac{M-w}{3}\right)$, because it provides the two retailers higher profits than any other equilibrium. Note that in this case, total order quantity is strictly less than $K$. The analysis of scenario (ii) is similar to (i).

Theorem 3 also shows that both retailers may inflate their orders under fixed factor allocation, relative to the order pair $\left(\frac{M-w}{3}, \frac{M-w}{3}\right)$ under sufficiently large capacity. This happens when the wholesale price is medium. For example, under conditions that $1 / 2 \leq \alpha \leq 4 / 5$ and $M-(2-\alpha) K<w<w^{c}(\alpha)$, an order in equilibrium $[\beta, K] \times[\hat{\alpha}, K]$ is strictly larger than $\frac{M-w}{3}$. In such cases, the supplier's capacity is sold out and retailer 1 maximizes her profit when she receives an allocation of $\alpha K$ in equilibrium ordering. Specifically, retailer 1 will inflate her order to guarantee an allocation of $\alpha K$, which induces retailer 2 to also inflate her order to guarantee an allocation of $(1-\alpha) K$.

\section{SUPPLIER'S DECISION}

In this section, we consider how the supplier, the Stackelberg game leader, determines his wholesale price to maximize his own profit. Theorem 3 illustrates how the two retailers react to the change in wholesale price, and thus it is straightforward to determine the supplier's optimal wholesale price. As noted in section 
Figure 1: Supplier's profit as a function of market size $M$.

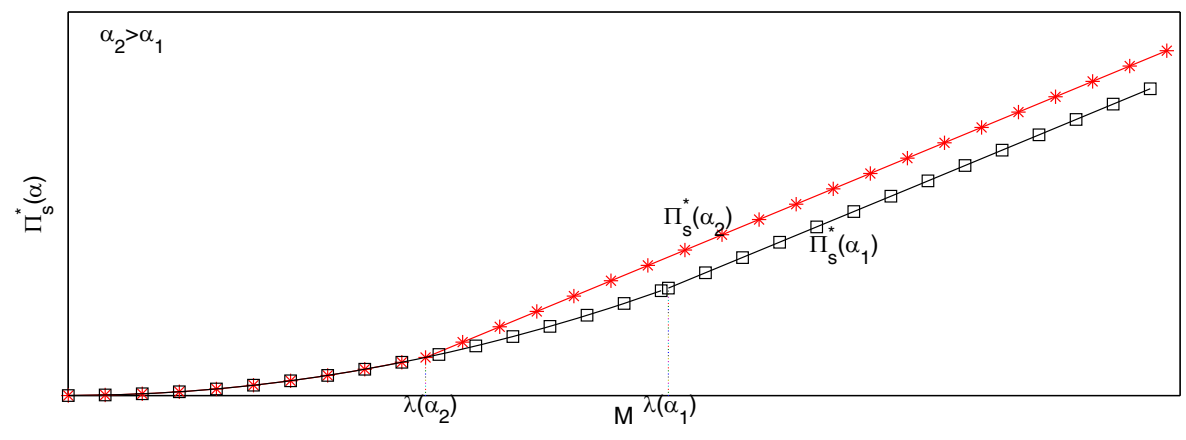

"Nash Equilibrium," for $w<w^{c}(\alpha)$, total order quantity in the nondominated Nash equilibrium equals total capacity $K$, and thus the total sale to the retailers is also $K$. In this case, the supplier's profit is $w K$. On the other hand, if $w \geq w^{c}(\alpha)$, then the total quantity ordered is less than the supplier's capacity, because the total order quantity $2(M-w) / 3$ is strictly less than $K$. Consequently, the supplier's maximum profit $\Pi_{s}^{*}(\alpha)$, as a function of the allocation factor $\alpha$, can be expressed as

$$
\Pi_{s}^{*}(\alpha)=\max \left\{\max _{w \in\left(0, w^{c}(\alpha)\right)} w K ; \max _{w \in\left[w^{c}(\alpha), M\right)} \frac{2(M-w) w}{3}\right\} .
$$

For notational simplicity, let $\lambda(\alpha)=3 K-3 \sqrt{\sqrt{(9 \alpha-4) \alpha}+1-3 \alpha} K$, which have the following properties.

\section{Lemma 3:}

(i) $\lambda(\alpha)$ is decreasing in $\alpha$.

(ii) If $M>\lambda(\alpha)$, then the supplier's optimal profit $\Pi_{s}^{*}(\alpha)=w^{c}(\alpha) K$, which is increasing in $\alpha$.

Theorem 4: If $0<M \leq \lambda(\alpha)$, then the optimal wholesale price is $w^{*}(\alpha)=M / 2$ and the supplier's maximum profit is $\Pi_{s}^{*}(\alpha)=M^{2} / 6$; otherwise, i.e., $M>\lambda(\alpha)$, the optimal wholesale price is $w^{*}(\alpha)=w^{c}(\alpha)^{-}$(which is greater than 0 for $\lambda(\alpha)>$ $\gamma(\alpha))$ and the supplier's maximum profit is $\Pi_{s}^{*}(\alpha)=w^{c}(\alpha) K$.

In Theorem 4, the term $w^{c}(\alpha)^{-}$means that the supplier should choose his wholesale price less than but as close as possible to $w^{c}(\alpha)$. This is due to the discontinuity of total order quantity as a function of the wholesale price when the wholesale price equals $w^{c}(\alpha)$.

Theorem 4 indicates that for any fixed factor $\alpha$, as the market size $M$ increases, the supplier's profit increases continuously, which is shown by Figure 1. This is intuitive, because $M$ represents the market potential of the supplier's product. What is not so intuitive is that as $M$ increases, the optimal wholesale price is 
Figure 2: Supplier's optimal wholesale price as a function of market size $M$.

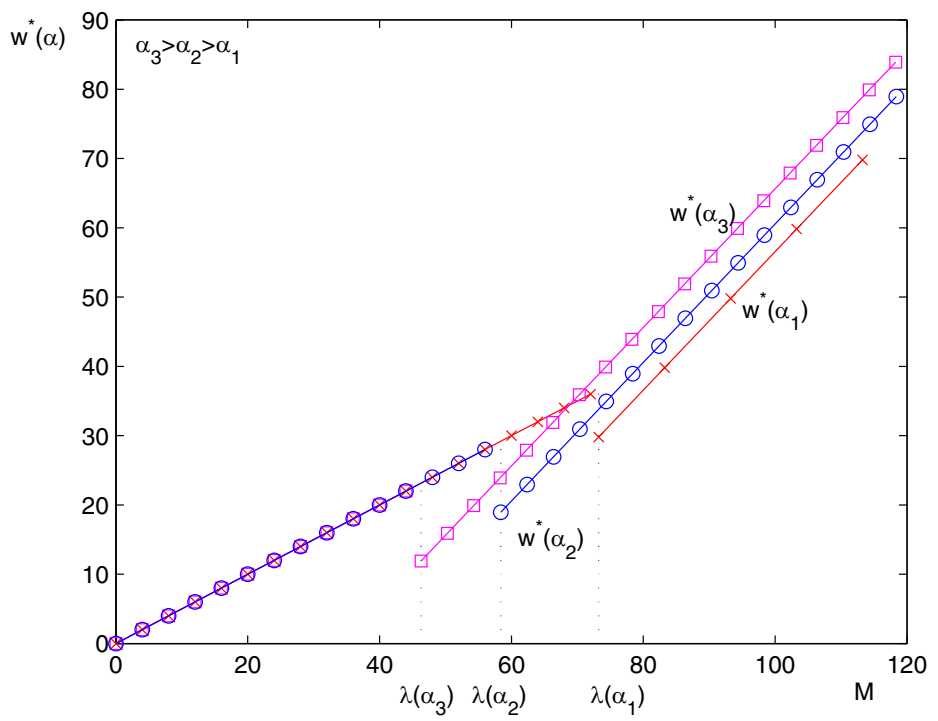

Table 1: Supply chain members' optimal decisions and profits.

\begin{tabular}{lll}
\hline Item & \multicolumn{1}{c}{$M \leq \lambda(\alpha)$} & \multicolumn{1}{c}{$M>\lambda(\alpha)$} \\
\hline$w^{*}$ & $\left.w^{*}(\alpha)\right)=M / 2$ & $w^{*}(\alpha)=(M-\gamma(\alpha))^{-}$ \\
$m_{1}^{*}$ & $m_{1}^{*}=M / 6$ & $m_{1}^{*} \geq K-m_{2}^{*}$ \\
$m_{2}^{*}$ & $m_{2}^{*}=M / 6$ & $m_{2}^{*} \geq K-m_{1}^{*}$ \\
$\Pi_{s}^{*}(\alpha)$ & $\Pi_{s}^{*}(\alpha)=M^{2} / 6$ & $\Pi_{s}^{*}(\alpha)=(M-\gamma(\alpha)) K$ \\
$\Pi_{1}^{*}(\alpha)$ & $\Pi_{1}^{*}(\alpha)=M^{2} / 36$ & $\Pi_{1}^{*}(\alpha)=(\gamma(\alpha)-K) \alpha K$ \\
$\Pi_{2}^{*}(\alpha)$ & $\Pi_{2}^{*}(\alpha)=M^{2} / 36$ & $\Pi_{2}^{*}(\alpha)=(\gamma(\alpha)-K)(1-\alpha) K$ \\
$\Pi_{r}^{*}(\alpha)$ & $\Pi_{r}^{*}(\alpha)=M^{2} / 18$ & $\Pi_{r}^{*}(\alpha)=(\gamma(\alpha)-K) K$ \\
$\Pi_{s c}^{*}(\alpha)$ & $\Pi_{s c}^{*}(\alpha)=2 M^{2} / 9$ & $\Pi_{s c}^{*}(\alpha)=(M-K) K$ \\
\hline
\end{tabular}

not necessarily increasing. When the market size $M$ is relatively small, the supplier chooses his wholesale price to induce retail demand, which is below capacity, and thus purposely allows certain capacity to go unused, and when the market size $M$ exceeds a threshold level, i.e., $\lambda(\alpha)$, the supplier suddenly reduces his wholesale price to achieve full capacity utilization. Therefore, behind the continuously increasing supplier profit as $M$ increases is a sudden drop in wholesale price, a sudden increase in total retail order quantity, and a transition from excess capacity to $100 \%$ capacity utilization, as shown in Figure 2.

From Theorems 3 and 4, we can locate supply chain members' optimal decisions and their corresponding profits under different market sizes, as in Table 1. Table 1 illustrates that if $M \leq \lambda(\alpha)$, then the optimal wholesale price is $w^{*}(\alpha)=$ $M / 2$ and the supplier's maximum profit is $\Pi_{s}^{*}(\alpha)=M^{2} / 6$, and the retailers order 
$m_{1}^{*}=m_{2}^{*}=\frac{M-w^{*}}{3}=M / 6$ and receive profits $\Pi_{1}^{*}(\alpha)=\Pi_{2}^{*}(\alpha)=\frac{M^{2}}{36}$. In this scenario, total order quantity is strictly less than $K$, i.e., the supplier maintains a capacity surplus to maximize his profit. On the other hand, under condition $M>\lambda(\alpha)$, the optimal wholesale price is $w^{*}(\alpha)=(M-\gamma(\alpha))^{-}$and the supplier's maximum profit is $\Pi_{s}^{*}(\alpha)=w^{c}(\alpha) K$, and the two retailers' order sizes satisfy $m_{1}^{*}+m_{2}^{*} \geq K$ and they receive allocations $g_{1}\left(m_{1}^{*}, m_{2}^{*}\right)=\alpha K$ and $g_{2}\left(m_{1}^{*}, m_{2}^{*}\right)=(1-\alpha) K$, respectively. Correspondingly, retailers 1 and 2 obtain profits (from Equations (8) and (9)) $\Pi_{1}^{*}(\alpha)=(\gamma(\alpha)-K) \alpha K$ and $\Pi_{2}^{*}(\alpha)=(\gamma(\alpha)-K)(1-\alpha) K$, respectively.

\section{IMPACTS OF FIXED FACTOR $\alpha$}

In this section, we investigate how the fixed factor $\alpha$ affects supply chain member decisions. Recall that the fixed factor allocation offers priority to retailer 1 . In case the two retailers' total order size exceeds the supplier's available capacity, retailer 1 receives an allocation of $\max \left\{\alpha K, K-m_{2}\right\}$ if she orders at least $\alpha K$; otherwise, she receives an allocation as she orders. Then, the remaining capacity is allocated to retailer 2.

Since the supplier is a game leader making wholesale price decision that directly decides retailers' equilibrium order quantity. We first study the effects of $\alpha$ on supplier's decision. Recall that in Theorem $4, \lambda(\alpha)$ is a threshold of the market size that determines supplier's optimal wholesale price, and by transformation, we can get $M=\lambda(\alpha)$ is equivalent to $\alpha=\frac{\left(\frac{M}{K}\right)^{2}}{54} \frac{\left(\frac{M}{K}-6\right)^{2}}{\frac{G M}{K}-6-\left(\frac{M}{K}\right)^{2}}$ when $M \leq 3 K$. For notational simplicity, it is useful to introduce the following: $u=\frac{M}{K}$ and $\tau=$ $\frac{u^{2}}{54} \frac{(u-6)^{2}}{6 u-6-u^{2}}$. Here, $u$ is the ratio of market size to capacity. And the value of $\tau$ serves as a threshold of $\alpha$, where $\alpha \leq \tau$ is equivalent to $M \leq \lambda(\alpha)$ and $\alpha>\tau$ is equivalent to $M>\lambda(\alpha)$ under condition $u \leq 3$. Besides, for $u>3, M>\lambda(\alpha)$ for any $\alpha \in\left[\frac{1}{2}, 1\right]$. Then, with market size and capacity exogenously given, the supplier's optimal decision relies on the value of $\alpha$. As $\alpha$ belongs to interval $\left[\frac{1}{2}, 1\right]$, it is necessary to find out how $\tau$ values with $u \leq 3$. Figure 3 depicts the relationship between $\tau$ and $u$. Figure 3 shows that for $u \in\left(u_{0}, u_{1}\right), \tau>1$; for $u \in\left[u_{1}, u_{2}\right], \tau \in\left[\frac{1}{2}, 1\right]$. Here, $u_{0}=3-\sqrt{3}, u_{1} \approx 1.5425, u_{2}=3$.

Now by Theorem 4 and the definitions of $u$ and $\tau$, we can precisely express the supplier's optimal wholesale price decision and profit in terms of fixed factor $\alpha$, as in the following theorem.

\section{Theorem 5:}

(i) $M<u_{1} K$. We have $M<\lambda(\alpha)$ for any $\alpha$. Thus, the optimal wholesale price is $w^{*}(\alpha)=M / 2$ and the supplier's maximum profit is $\Pi_{s}^{*}(\alpha)=$ $M^{2} / 6$.

(ii) $M \in\left[u_{1} K, u_{2} K\right]$. If $\alpha \in\left[\frac{1}{2}, \tau\right]$, then $M \leq \lambda(\alpha), w^{*}(\alpha)=M / 2$, and $\Pi_{s}^{*}(\alpha)=M^{2} / 6$; if $\alpha \in(\tau, 1]$, then $M>\lambda(\alpha), w^{*}(\alpha)=w^{c}(\alpha)^{-}$, and $\Pi_{s}^{*}(\alpha)=w^{c}(\alpha) K$.

(iii) $M>u_{2} K$. We have $M>\lambda(\alpha)$ for any $\alpha$. Thus, the optimal wholesale price is $w^{*}(\alpha)=w^{c}(\alpha)^{-}$and the supplier's maximum profit is $\Pi_{s}^{*}(\alpha)=$ $w^{c}(\alpha) K$. 
Figure 3: Relationship between $\tau$ and $u$.

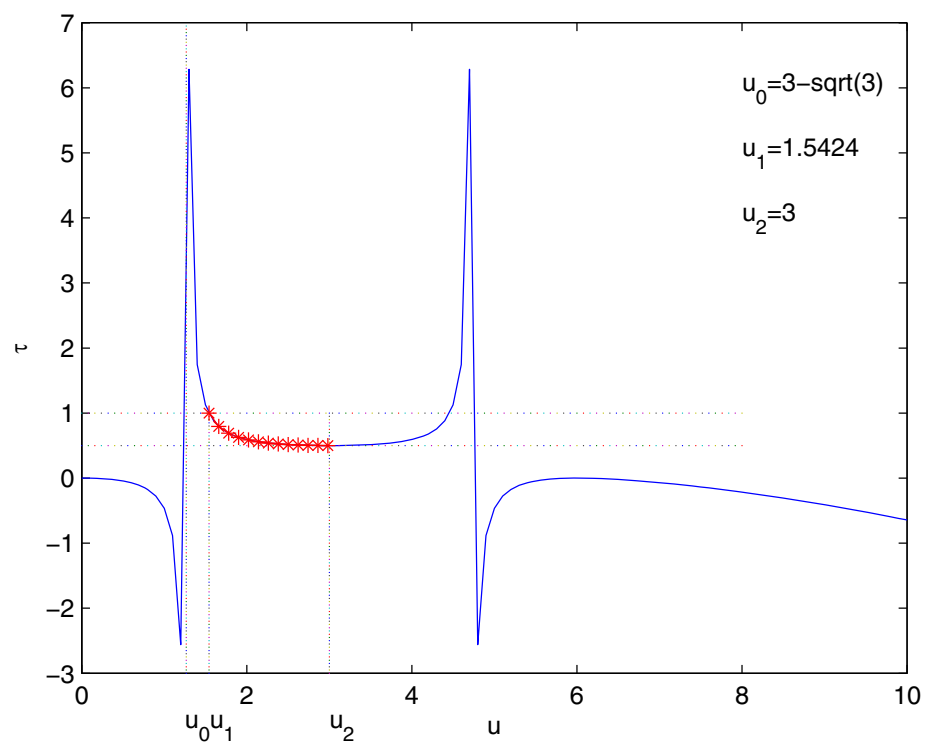

Table 2: Optimal decisions and profits in small or large market.

\begin{tabular}{lll}
\hline Item & \multicolumn{1}{c}{$M<u_{1} K$} & \multicolumn{1}{c}{$M>u_{2} K$} \\
$w^{*}$ & $w^{*}(\alpha)=M / 2$ & \multicolumn{1}{c}{$w^{*}(\alpha)=w^{c}(\alpha)^{-}$} \\
\hline$\Pi_{s}^{*}(\alpha)$ & $\Pi_{s}^{*}(\alpha)=M^{2} / 6$ & $\Pi_{s}^{*}(\alpha)=w^{c}(\alpha) K$ \\
$m_{1}^{*}$ & $m_{1}^{*}=\frac{M-w^{*}}{M \frac{3}{6}}=\frac{M}{6}$ & $m_{1}^{*} \in[\alpha K, K]$ \\
$m_{2}^{*}$ & $m_{2}^{*}=\frac{M-w^{*}}{3}=\frac{M}{6}$ & $m_{2}^{*} \in[\hat{\alpha}, K]$ \\
$\Pi_{1}^{*}(\alpha)$ & $\Pi_{1}^{*}(\alpha)=M^{2} / 36$ & $\Pi_{1}^{*}(\alpha)=(\gamma(\alpha)-K) \alpha K$ \\
$\Pi_{2}^{*}(\alpha)$ & $\Pi_{2}^{*}(\alpha)=M^{2} / 36$ & $\Pi_{2}^{*}(\alpha)=(\gamma(\alpha)-K)(1-\alpha) K$ \\
$\Pi_{r}^{*}(\alpha)$ & $\Pi_{r}^{*}(\alpha)=M^{2} / 18$ & $\Pi_{r}^{*}(\alpha)=(\gamma(\alpha)-K) K$ \\
$\Pi_{s c}^{*}(\alpha)$ & $\Pi_{s c}^{*}(\alpha)=2 M^{2} / 9$ & $\Pi_{s c}^{*}(\alpha)=(M-K) K$ \\
\hline
\end{tabular}

We define three types of market based on market size. Specifically, we refer to the market as small, medium, and large under conditions $M<u_{1} K$, $M \in\left[u_{1} K, u_{2} K\right]$ and $M>u_{2} K$, respectively. With Theorem 5, we characterize the optimal decisions and profits of the supply chain members in Tables 2 and 3. Specifically, Table 2 characterizes the scenarios that the market size is either relatively small or sufficiently large, and Table 3 covers the remaining scenario with medium market size.

From Table 2, when market size is small but can still be larger than the capacity level $K\left(M<u_{1} K, u_{1} \approx 1.5\right)$, the supplier always sets his optimal wholesale price as $M / 2$, which maintains a strict capacity surplus to maximize his profit at $M^{2} / 6$. If the market size is less than $K$, then this result is consistent with our intuition. It is interesting that the supplier still chooses to sell less than $K$ even 
Table 3: Optimal decisions and profits in medium market.

\begin{tabular}{lll}
\hline Item & \multicolumn{1}{c}{$\alpha \leq \tau$} & \multicolumn{1}{c}{$\alpha>\tau$} \\
\hline$w^{*}$ & $w^{*}(\alpha)=M / 2$ & $w^{*}(\alpha)=w^{c}(\alpha)^{-}$ \\
$\Pi_{s}^{*}(\alpha)$ & $\Pi_{s}^{*}(\alpha)=M^{2} / 6$ & $\Pi_{s}^{*}(\alpha)=w^{c}(\alpha) K$ \\
$m_{1}^{*}$ & $m_{1}^{*}=\frac{M-w^{*}}{M}=\frac{M}{6}$ & $m_{1}^{*} \in[\alpha K, K]$ \\
$m_{2}^{*}$ & $m_{2}^{*}=\frac{M-w^{*}}{3}=\frac{M}{6}$ & $m_{2}^{*} \in[\hat{\alpha}, K]$ \\
$\Pi_{1}^{*}(\alpha)$ & $\Pi_{1}^{*}(\alpha)=M^{2} / 36$ & $\Pi_{1}^{*}(\alpha)=(\gamma(\alpha)-K) \alpha K$ \\
$\Pi_{2}^{*}(\alpha)$ & $\Pi_{2}^{*}(\alpha)=M^{2} / 36$ & $\Pi_{2}^{*}(\alpha)=(\gamma(\alpha)-K)(1-\alpha) K$ \\
$\Pi_{r}^{*}(\alpha)$ & $\Pi_{r}^{*}(\alpha)=M^{2} / 18$ & $\Pi_{r}^{*}(\alpha)=(\gamma(\alpha)-K) K$ \\
$\Pi_{s c}^{*}(\alpha)$ & $\Pi_{s c}^{*}(\alpha)=2 M^{2} / 9$ & $\Pi_{s c}^{*}(\alpha)=(M-K) K$ \\
\hline
\end{tabular}

when $M \geq K$ (slightly). In this scenario, the two retailers both order $M / 6$ and gain the corresponding profit $M^{2} / 36$, and thus the supply chain's total profit is $2 M^{2} / 9$. As a result, the allocation factor $\alpha$ does not affect the supplier's decisions when the market size is small, which can be explained as follows. The fixed factor allocation provides priority to retailer 1 , with $\alpha \geq 1 / 2$. When the market size is small, retailer 1 orders $(M-w) / 3$ (her desired quantity), which is less than the quantity reserved for her $(\alpha K)$. Consequently, the remaining capacity is greater than retailer 2's optimal order quantity. Therefore, the fixed factor $\alpha$ does not affect the supplier and the retailers' decisions.

When market size is sufficiently large $\left(M>u_{2} K, u_{2}=3\right)$, the retailers will order as much as possible so long as the marginal profit is positive. Bearing this in mind, the supplier sets an optimal wholesale price that is increasing in $\alpha$ and sells out his full capacity, and consequently his optimal profit is increasing in $\alpha$. That is, it is optimal for the supplier to set $\alpha=1$ if $\alpha$ is a decision variable. Note that retailer 1's order quantity and allocated quantity are both increasing in $\alpha$ and retailer 2's allocated quantity is decreasing in $\alpha$. The supplier always faces beyond-capacity-demand and sells out his capacity. These results are consistent with our intuition. The profits of retailers 1 and 2 are both decreasing in $\alpha$. As in the case $M>u_{2} K$, the supply chain's total profit does not vary with $\alpha$. That is, varying $\alpha$ only reallocates the profit among these members of the supply chain.

\section{Remark 1:}

(i) When market size is sufficiently small, each supply chain member's profit is independent of the allocation factor $\alpha$.

(ii) When market size is sufficiently large, the supplier prefers a large allocation factor ( $\alpha=1$, i.e., lexicographic allocation) and his profit is related to market size, but the two retailers both prefer a small allocation factor $(\alpha=1 / 2)$ and their profits are independent of market size.

Thus far, we have analyzed cases where the market size is either sufficiently small or sufficiently large. Next, we investigate the scenario with medium market size, i.e., $M \in\left[u_{1} K, u_{2} K\right]$. By Theorem 5, we immediately obtain the results 
Table 4: Supply chain members' optimal decisions when $\alpha$ approaches $\tau$.

\begin{tabular}{llll}
\hline Item & $\alpha=\tau$ & \multicolumn{1}{c}{$\alpha=\tau^{+}$} & \multicolumn{1}{c}{ Comparison } \\
\hline$w^{*}$ & $M / 2$ & $w^{c}(\tau)^{-}$ & $M / 2>w^{c}(\tau)^{-}$ \\
$\Pi_{s}^{*}$ & $M^{2} / 6$ & $(M-\gamma(\tau)) K$ & $M^{2} / 6=(M-\gamma(\tau)) K$ \\
$g_{1}\left(m_{1}^{*}, m_{2}^{*}\right)$ & $\frac{M}{6}$ & $\tau K$ & $\frac{M}{6}<\tau K$ \\
$g_{2}\left(m_{1}^{*}, m_{2}^{*}\right)$ & $\frac{M}{6}$ & $(1-\tau) K$ & if $M \in\left[u_{1} K,(6-3 \sqrt{2}) K\right), \frac{M}{6}>$ \\
& & & $(1-\tau) K ;$ if \\
& & & $M \in\left[(6-3 \sqrt{2}) K, u_{2} K\right]$, \\
& & & $\frac{M}{6} \leq(1-\tau) K$ \\
$\Pi_{1}^{*}$ & $M^{2} / 36$ & $(\gamma(\tau)-K) \tau K$ & $M^{2} / 36<(\gamma(\tau)-K) \tau K$ \\
$\Pi_{2}^{*}$ & $M^{2} / 36$ & $(\gamma(\tau)-K)$ & if $M \in\left[u_{1} K, 1.8351 K\right), M^{2} / 36>$ \\
& & $(1-\tau) K$ & $(\gamma(\tau)-K)(1-\tau) K ;$ if \\
& & & $M \in\left[1.8351 K, u_{2} K\right]$, \\
$\Pi_{s c}$ & & & $M^{2} / 36 \leq(\gamma(\tau)-K)(1-\tau) K$ \\
\hline
\end{tabular}

shown in Table 3. To better understand the results in Table 3, we need the following lemma.

Lemma 4: Under condition that $M \in\left[u_{1} K, u_{2} K\right]$ (i.e., $u \in\left[u_{1}, u_{2}\right]$, which implies that $\left.\tau \in\left[\frac{1}{2}, 1\right]\right)$, we provide the comparisons in Table 4 in terms of the supply chain members' optimal decisions when $\alpha=\tau$ and $\alpha=\tau^{+}\left(\tau^{+}\right.$means that $\alpha$ values more than but as close as possible to $\tau$ ).

Table 4 in Lemma 4 shows changes of supplier's optimal wholesale price, retailers' allocated quantity, and respective supply chain members' profits when $\alpha$ approximates the threshold point $\tau$. With Lemma 4, we can characterize the scenario with medium market size, as illustrated in Figures 4-10, where we show different quantities of interest as a function of $\alpha$. We show the values for three different market sizes $M_{1}, M_{2}, M_{3}$. The values $\tau_{1}, \tau_{2}, \tau_{3}$ indicate the $\tau$ values that correspond to the three different market sizes, respectively. Figure 4 shows how the supplier's optimal wholesale price changes with $\alpha$ at medium market size. At the point $\alpha=\tau$, the optimal wholesale price has a sudden drop, which implies the selling of more capacity, and then is increasing in $\alpha$. Furthermore, if $M \in\left[u_{1} K,(9-3 \sqrt{5}) K\right)$ (the case of $\left.M_{1}\right)$, then the optimal wholesale price when $\alpha>\tau$ is less than that when $\alpha \leq \tau(M / 2)$. Then, Figures 5 and 6 show how two retailers' allocated quantities in equilibrium ordering change with $\alpha$ at medium market size corresponding to the optimal wholesale price. Retailer 1's allocated quantity jumps at the point $\alpha=\tau$, then is increasing in proportion with $\alpha$. It indicates that when $\alpha>\tau$, retailer 1 will always order more than $\alpha K$ regardless of the increase of optimal wholesale price and is also allocated that quantity. Different from retailer 1, retailer 2's allocated quantity experiences a drop or jump at the point $\alpha=\tau$ that depends on the market size $(M<(6-3 \sqrt{2}) K$ or $M>(6-3 \sqrt{2}) K)$, then is decreasing in proportion with $(1-\alpha)$. However, in spite of a low allocated quantity, retailer 2 always orders more than $(1-\alpha) K$ to reach an equilibrium. 
Figure 4: Optimal wholesale price in medium market.

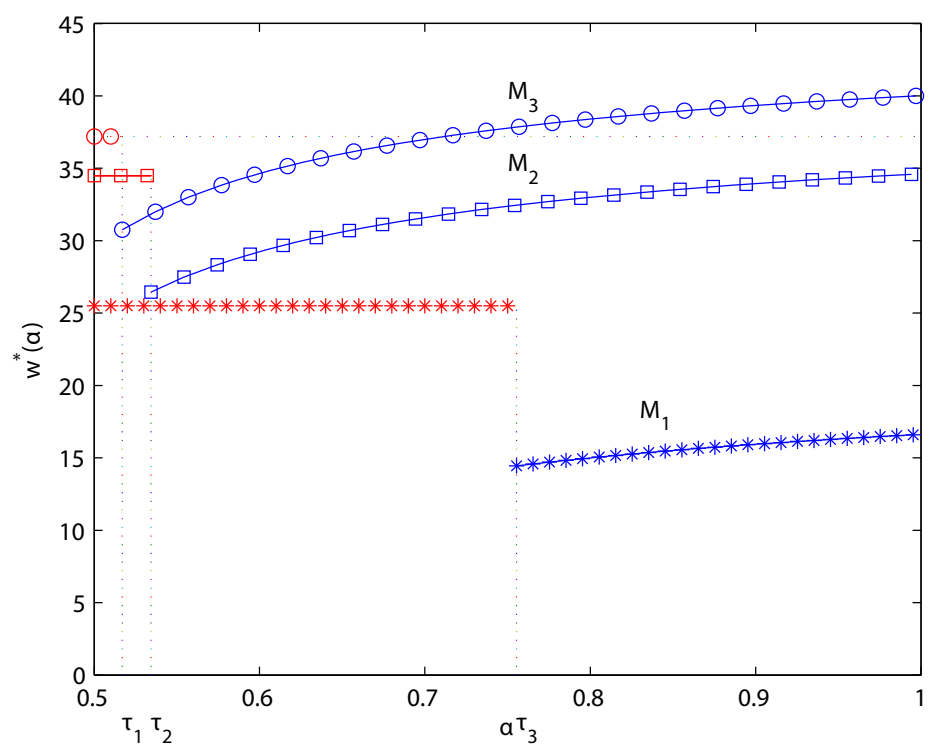

Figures 7-10 show how the supplier members' optimal profits change with $\alpha$ at medium market size. The supplier's profit is increasing in $\alpha$. Although the supplier's optimal wholesale price has a sudden drop at the point $\alpha=\tau$, the supplier's optimal profit increases continuously with $\alpha$. Besides, both retailers' profits have a sudden jump at the point $\alpha=\tau$ when the market size is relatively large $\left(M \in\left[1.8351 K, u_{2} K\right]\right)$, while both they are decreasing in $\alpha$ when $\alpha>\tau$ because of the increasing optimal wholesale price. Intuitively, the two retailers both prefer allocation factor $\alpha=\tau^{+}$because that the supplier adjusts her wholesale price according to the value of $\alpha$. It is interesting to see that the supply chain profit jumps at the point $\alpha=\tau$ and then maintains a constant value for any $\alpha \in(\tau, 1]$ at the given market size. The reason is when $\alpha>\tau$, the supplier can always sell out all its capacity at its optimal wholesale price.

Remark 2: In medium market, the supplier prefers an allocation factor to be as large as possible $(\alpha=1)$, but the two retailers prefer allocation factor $\alpha=\tau^{+}$, which is related to market size $M$ and capacity level $K\left(M \in\left[1.8351 K, u_{2} K\right]\right)$. The supply chain achieves the same maximum profit in the region $\alpha \in(\tau, 1]$.

\section{COMPARISON WITH CENTRALIZED SUPPLY CHAIN}

In this section, we compare the supply chain profit under fixed factor allocation with the profit of a centralized supply chain.

Let $\Pi_{s c}^{c *}$ denote the maximum supply chain profit under centralized decisions. To obtain $\Pi_{s c}^{c *}$, let $q$ be the total quantity sold. The supply chain profit can be 
Figure 5: Retailer 1's equilibrium allocated quantity in medium market.

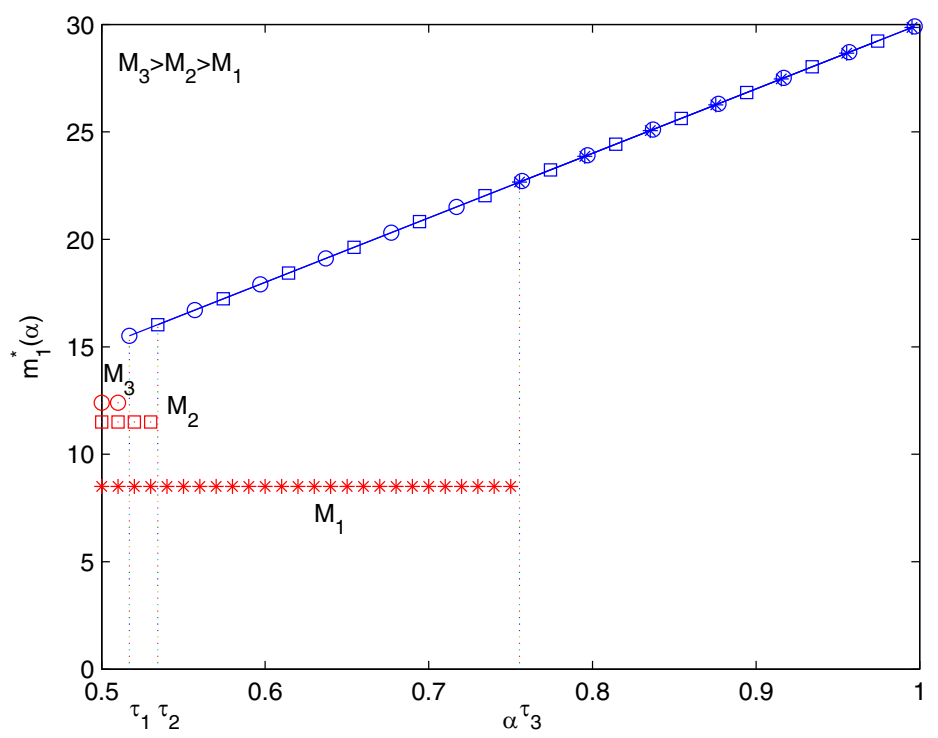

Table 5: Comparison with centralized supply chain.

\begin{tabular}{llll}
\hline$M$ & \multicolumn{1}{c}{ Decentralized system } & Centralized system & Comparison \\
\hline$M \in\left[0, u_{1} K\right)$ & $\Pi_{s c}^{*}(\alpha)=2 M^{2} / 9$ & $\Pi_{s c}^{c *}=\frac{M^{2}}{4}$ & $\Pi_{s c}^{*}(\alpha)<\Pi_{s c}^{c *}$ \\
$M \in\left(u_{1} K, 2 K\right]$ & $\Pi_{s c}^{*}(\alpha)=2 M^{2} / 9$ for $\alpha \leq \tau$ & $\Pi_{s c}^{c *}=\frac{M^{2}}{4}$ & $\Pi_{s c}^{*}(\alpha)<\Pi_{s c}^{c *}$ \\
& $\Pi_{s c}^{*}(\alpha)=(M-K) K$ for $\alpha>\tau$ & $\Pi_{s c}^{c *}=\frac{M^{2}}{4}$ & $\Pi_{s c}^{*}(\alpha)<\Pi_{s c}^{c *}$ \\
$M \in\left(2 K, u_{2} K\right]$ & $\Pi_{s c}^{*}(\alpha)=2 M^{2} / 9$ for $\alpha \leq \tau$ & $\Pi_{s c}^{c *}=(M-K) K$ & $\Pi_{s c}^{*}(\alpha)<\Pi_{s c}^{c *}$ \\
& $\Pi_{s c}^{*}(\alpha)=(M-K) K$ for $\alpha>\tau$ & $\Pi_{s c}^{c *}=(M-K) K$ & $\Pi_{s c}^{*}(\alpha)=\Pi_{s c}^{c *}$ \\
$M \in\left(u_{2} K,+\infty\right)$ & $\Pi_{s c}^{*}(\alpha)=(M-K) K$ & $\Pi_{s c}^{c *}=(M-K) K$ & $\Pi_{s c}^{*}(\alpha)=\Pi_{s c}^{c *}$ \\
\hline
\end{tabular}

written as $\Pi_{s c}^{C}=(M-q) q$. Note that the optimal selling quantity $q^{*}=$ $\min \left\{K, \frac{M}{2}\right\}$. Thus, $\Pi_{s c}^{c *}=\frac{M^{2}}{4}$ for $M \leq 2 K$ and $\Pi_{s c}^{c *}=(M-K) K$ for $M>2 K$. The value $\Pi_{s c}^{c *}$ provides a benchmark for measuring the loss of efficiency in a decentralized supply chain using the fixed factor allocation, where the supplier chooses wholesale price to maximize his own value instead of the supply chain value. Table 5 illustrates the results of comparing supply chain profits obtained from decentralized and centralized supply chains.

An important observation from Table 5 is that the supply chain profit in the decentralized supply chain with fixed factor allocation is the same as in the centralized supply chain when $M>>K$, i.e., the supplier chooses his optimal wholesale price that coordinates the supply chain, i.e., obtains the same supply chain profit as in a centralized supply chain. Specifically, the optimal decision in the centralized system is to sell the whole capacity to the market when the 
Figure 6: Retailer 2's equilibrium allocated quantity in medium market.

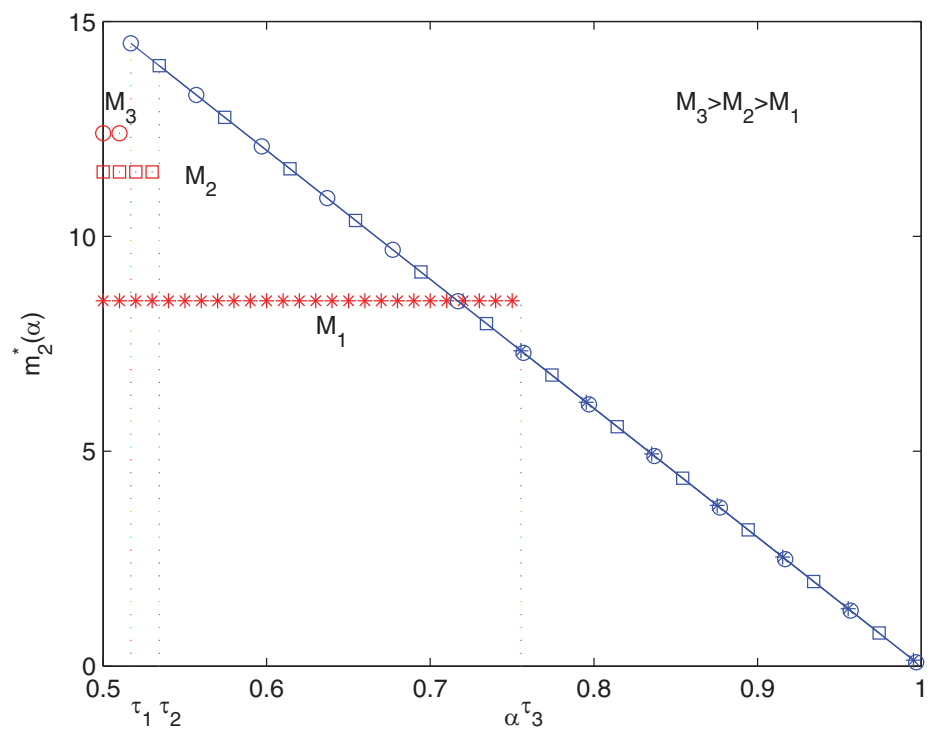

market size is large enough (roughly, $M>2 K$ ). In the decentralized system with two retailers' equilibrium order quantities in consideration, the supplier's optimal decision is to set a wholesale price to sell out the total capacity. Accordingly, under the manufacturer's selected wholesale price, the two retailers each orders as much as possible $(K)$ because of quantity competition. Consequently, each retailer is allocated her guaranteed quantity, i.e., $\alpha K$ by retailer 1 and $(1-\alpha) K$ by retailer 2 . As a result, the centralized and decentralized systems sell the same quantity $K$ and achieve the same supply chain profit $(M-K) K$.

Remark 3: The fixed factor allocation coordinates the supply chain when $M \in$ ( $\left.2 K, u_{2} K\right]$ with $\alpha>\tau$, and when $M>u_{2} K$ with $\alpha \geq 1 / 2$.

\section{COMPARISON WITH OTHER ALLOCATIONS}

Note that fixed factor allocation degenerates to lexicographic allocation when $\alpha=1$. We next compare fixed factor allocation with proportional allocation. We first cite a result from Chen, $\mathrm{Li}$, \& Zhang (2013), as in the following proposition.

Proposition 1: (Chen, Li, \& Zhang, 2013) Under proportional allocation,

(i) if $M \leq 3(2-\sqrt{2}) K$, then the supplier's optimal wholesale price is $w^{*}=$ $M / 2$, achieving a profit of $\Pi_{s}^{*}=M^{2} / 6$;

(ii) if $M>3(2-\sqrt{2}) K$, then the supplier's optimal wholesale price is $w^{*}=(M+3 K-3 \sqrt{2} K)^{-}$, achieving a profit of $\Pi_{s}^{*}=K(M+3 K-$ $3 \sqrt{2} K)^{-}$. 
Figure 7: Supplier's optimal profit in medium market.

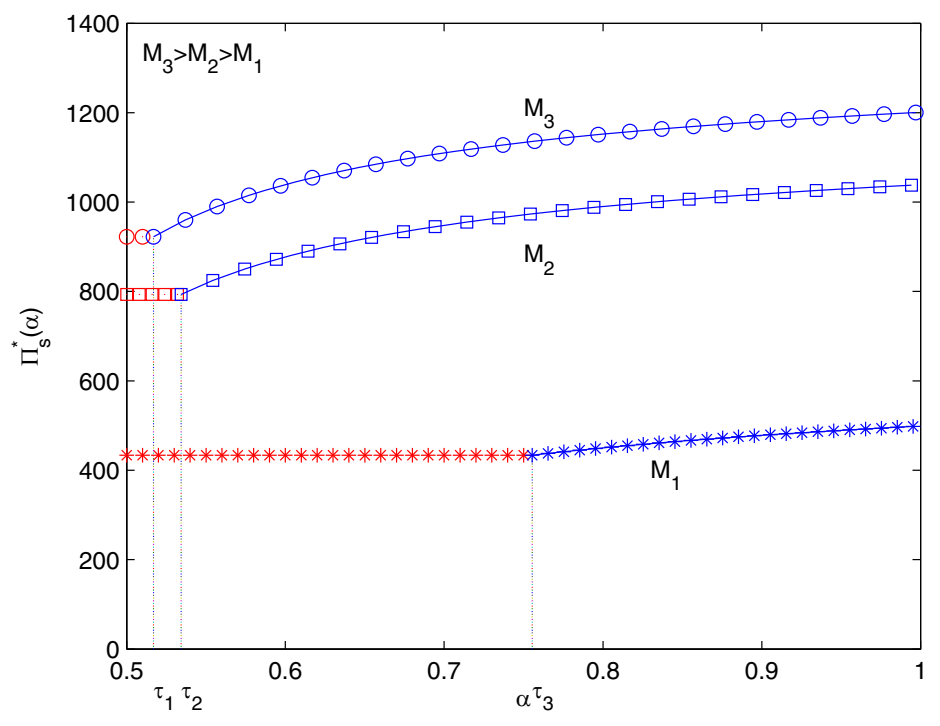

Figure 8: Retailer 1's profit in medium market.

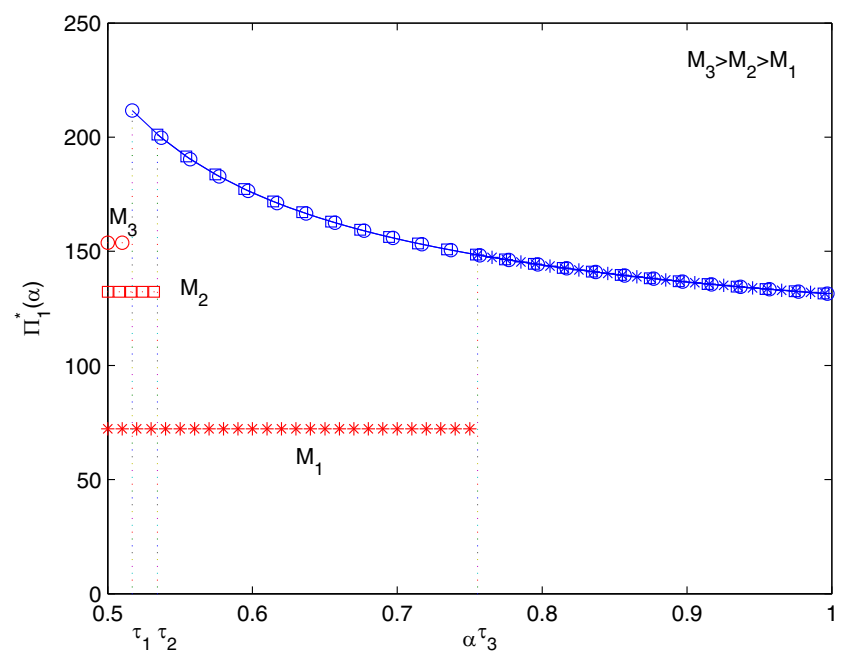

With Theorem 4 and Proposition 1, we can immediately obtain the following result from the view of the supplier and supply chain.

Theorem 6: If $\alpha=\sqrt{2} / 2$, then

(i) the supplier and supply chain will obtain the same profit under fixed factor and proportional allocations; 
Figure 9: Retailer 2's profit in medium market.

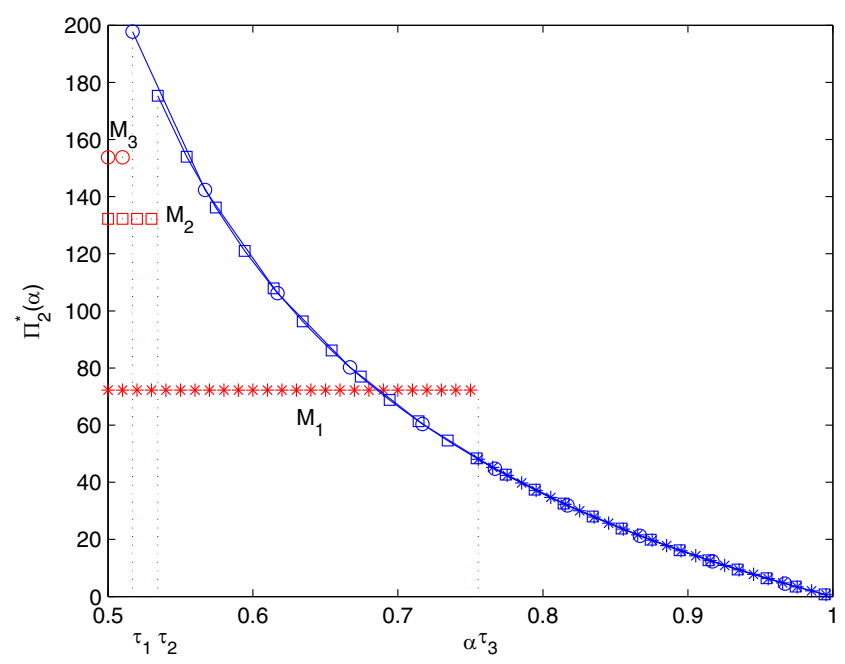

Figure 10: Supply chain profit in medium market.

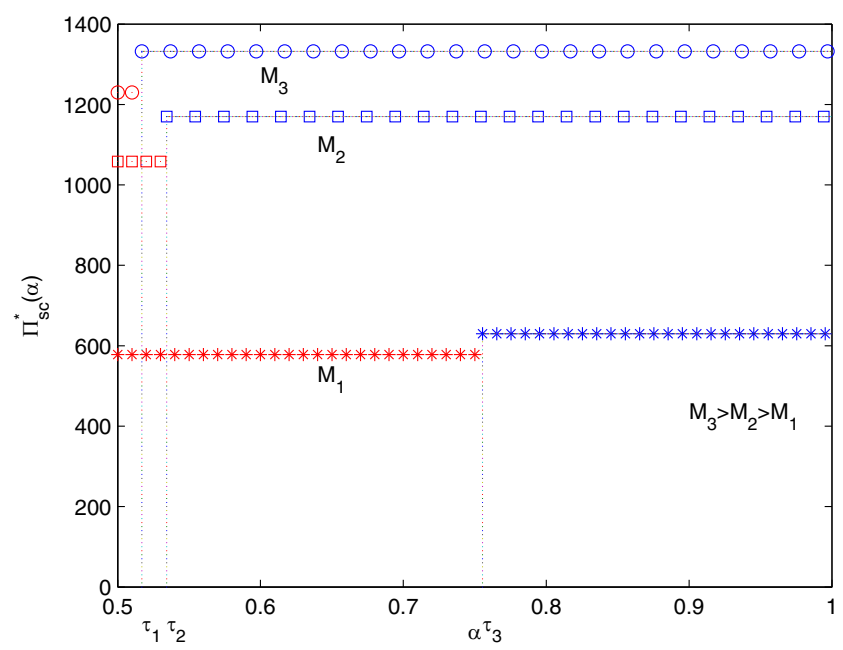

(ii) in equilibrium, under fixed factor allocation, retailer 1 gains more profit, retailer 2 gains less profit, and the total profit of the two retailers is the same, compared with under proportional allocation.

Theorem 6 indicates that fixed factor allocation is a general rule that is equivalent to proportional allocation with $\alpha=\sqrt{2} / 2$ in terms of the supplier's and the supply chain profits, under the supplier's optimal wholesale price decision and the retailers' equilibrium ordering. Next, we compare fixed factor, lexicographic, 
Figure 11: Supply chain profits under three allocations, as a function of $\alpha$.

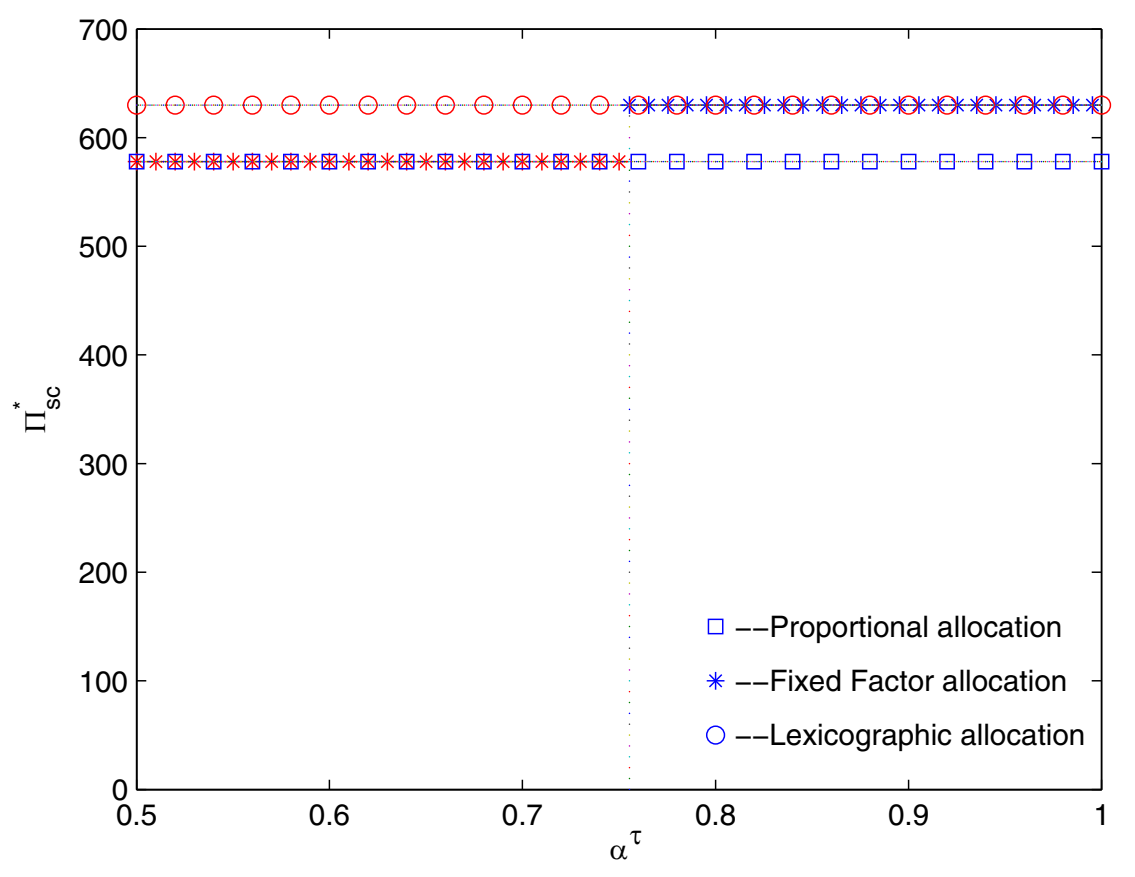

and proportional allocations numerically in terms of supply chain profit. There are three cases characterized by different levels of market size $M$ in terms of comparing the supply chain profit under the three allocations. Specifically, (1) if $M<u_{1} K$, then the supply chain profits are the same under the three allocations, which are $2 M^{2} / 9$; (2) if $u_{1} K \leq M \leq 3(2-\sqrt{2}) K$, then the supply chain obtains the same profit $(M-K) K$ under fixed factor (with $\alpha>\tau)$ and lexicographic allocations, which is larger than that of $2 M^{2} / 9$ under proportional allocation, while when $\alpha<\tau$, the supply chain gains the same profit under fixed factor and proportional allocations; (3) if $M>3(2-\sqrt{2}) K$, then the three allocations induce the same supply chain profit $(M-K) K$. Comparisons under case $2\left(u_{1} K \leq\right.$ $M<3(2-\sqrt{2}) K)$ are illustrated in Figure 11. The figure shows that the supply chain obtains more profit under fixed factor allocation than that under proportional allocation with market size at certain levels. And for a large range of $\alpha$, instead of single point $\alpha=1$, fixed factor allocation offers the same supply chain profit as lexicographic allocation does.

\section{OPTIMAL CAPACITY CHOICE}

In this section, we consider how to choose optimal capacity and optimal wholesale price from the view of the supplier. From Theorem 4, for each capacity level $K$, 
the supplier's optimal profit is

$$
\Pi_{s}^{*}(K)= \begin{cases}\max _{K}\left\{w^{c}(\alpha) K-c K\right\}, & \text { if } K \in\left[0, \frac{M}{3-3 \sqrt{\sqrt{(9 \alpha-4) \alpha}+1-3 \alpha}}\right), \\ \max _{K}\left\{\frac{M^{2}}{6}-c K\right\}, & \text { if } K \in\left[\frac{M}{3-3 \sqrt{\sqrt{(9 \alpha-4) \alpha}+1-3 \alpha}},+\infty\right),\end{cases}
$$

where $c$ is the cost for each capacity unit. To make the supplier's profit positive, we assume $M>c$.

Theorem 7: For the supplier, the optimal capacity is $K^{*}(\alpha)=\frac{M-c}{9 \alpha-3 \sqrt{(9 \alpha-4) \alpha}}$, and optimal wholesale price is $\left(\frac{M+c}{2}\right)^{-}$, which is independent of the allocation factor $\alpha$, and the corresponding optimal profit is $\frac{(M-c)^{2}}{2(9 \alpha-3 \sqrt{(9 \alpha-4) \alpha})}$.

From Theorem 7, we see that the supplier can achieve the maximum profit $\frac{(M-c)^{2}}{2(9 \alpha-3 \sqrt{(9 \alpha-4) \alpha})}$ by choosing capacity level $K^{*}(\alpha)=\frac{M-c}{9 \alpha-3 \sqrt{(9 \alpha-4) \alpha}}$ and wholesale price $\left(\frac{M+c}{2}\right)^{-}$. This optimal setting for the supplier induces that total order quantity from the two retailers be greater than $K^{*}$. That is, the capacity $\frac{M-c}{9 \alpha-3 \sqrt{(9 \alpha-4) \alpha}}$ is allocated in full.

\section{CONCLUDING REMARKS}

This article considers a supply chain consisting of one supplier and two competing retailers. The important components of our model are the supplier's limited production capacity and the Cournot competition between the retailers. Because of the capacity constraint, an allocation rule is needed for capacity allocation in case the retailers' total order size exceeds the supplier's available capacity. The fixed factor allocation rule is proposed, which first guarantees a certain proportion of capacity to a prioritized retailer, then the surplus capacity can be allocated to the other retailer, incorporating the ideas of proportional and lexicographic allocations. Under fixed factor allocation, with exogenously given capacity and market demand, the supplier should carefully choose the allocation factor and the wholesale price to maximize his profit, keeping in mind that retailers order in equilibrium to maximize their own individual profits.

Under fix factor allocation, we show that in both sufficiently small and sufficiently large markets, the allocation factor has no effects on the supply chain profit. However, in medium market, the supply chain profit is not affected by the allocation factor only when the factor is greater than a particular threshold, and accordingly, the retailers share the supply chain profit with the supplier depending on the value of the allocation factor. Also, we prove that retailers prefer an allocation factor different from that preferred by the supplier, but the preferred allocation factor values by both the retailers and the supplier are in the range that maximizes total supply chain profit. Furthermore, we show that the fixed factor allocation coordinates the supply chain when the market size is sufficiently large. We also compare fixed factor with proportional and lexicographic allocations, respectively. We show that fixed factor allocation can incorporate both lexicographic and proportional allocations from the perspective of the supplier and the supply chain. Moreover, the supply chain obtains more profit under fixed factor allocation 
than that under proportional allocation with market size at certain levels. And for a large range of $\alpha$, instead of single point $\alpha=1$, fixed factor allocation offers the same supply chain profit as lexicographic allocation does. Finally, we demonstrate how the supplier can optimize his capacity level and wholesale price under fixed factor allocation.

Our work can be extended in several ways. First, it would be interesting to consider asymmetric retailers with different market powers. Second, it would be valuable to study a case with more than two retailers. Third, it would be worthwhile to compare fixed factor allocation with other allocations, such as linear and uniform allocations. Finally, it would be helpful to study the gaming effect caused by fixed factor allocation and bounded rationality using laboratory experiments, as by Chen, $\mathrm{Su}$, and Zhao (2012) for proportional allocation.

\section{REFERENCES}

Blumenstein, R. (1996). Autos: How do you get a hot GMC Suburban? You wait for a computer to dole one out. Wall Street Journal, April 10, B1.

Cachon, G. P., \& Lariviere, M. A. (1999a). Capacity choice and allocation: Strategic behavior and supply chain performance. Management Science, 45, 10911108.

Cachon, G. P., \& Lariviere, M. A. (1999b). An equilibrium analysis of linear, proportional and uniform allocation of scarce capacity. IIE Transactions, 31, 835-849.

Cachon, G. P., \& Lariviere, M. A. (1999c). Capacity allocation using past sales: When to turn-and-earn. Management Science, 45, 685-703.

Chen, F., Li, J., \& Zhang, H. (2013). Managing downstream competition via capacity allocation. Production and Operations Management, 22(2), 426446.

Chen, Y., Su, X., \& Zhao, X. (2012). Modeling bounded rationality in capacity allocation games with the quantal response equilibrium. Management Science, 58(10), 1952-1962.

Chen, F., \& Zheng, Y. (1994). Lower bounds for multi-echelon stochastic inventory systems. Management Science, 40, 1426-1443.

Cho, S. H., \& Tang, C. (2014). Capacity allocation under retail competition: Uniform and competitive allocations. Operations Research, 62(1), 72-80.

Clark, A. J., \& Scarf, H. (1960). Optimal policies for a multi-echelon inventory problem. Management Science, 6, 475-490.

Deshpande, V., Cohen, M. A., \& Donohue, K. (2003). A threshold inventory rationing policy for service differentiated demand classes. Management Science, 49(6), 683-703.

Deshpande, V., \& Schwarz, L. B. (2002). Optimal capacity choice and allocation in decentralized supply chains. Working paper, Krannert School of Management, Purdue University, West Lafayette, Purdue.

Eppen, G., \& Schrage, L. (1981). Centralized ordering policies in multi-echelon systems with leadtimes and random demand. In L. B. Schwarz (Ed.), 
Multi-level production/inventory control systems: Theory and practice 16. Amsterdam: North-Holland, 51-67.

Federgruen, A., \& Zipkin, P. (1984). Allocation policies and cost approximations for multilocation production and inventory problems. Management Science, 30, 69-84.

Ha, A. Y. (1997). Inventory rationing in a make-to-stock production system with several demand classes and lost sales. Management Science, 43, 1093-1103.

Hall, N. G., \& Liu, Z. (2010). Capacity allocation and scheduling in supply chains. In J. J. Cochran (editor-in-chief), Wiley encyclopedia of operations research and management science. New York City, NY: John Wiley \& Sons, Inc., 576-584.

Hwang, S. L., \& Valeriano, L.L. (1992). Marketers and consumers get the jitters over severe shortages of nicotine patches. Wall Street Journal, May 22, B1.

Jonsson, H., \& Silver, E. (1987). Analysis of a two-echelon inventory-control system with complete redistribution. Management Science, 33, 215-227.

Liu, Z. (2012). Equilibrium analysis of capacity allocation with demand competition. Naval Research Logistics, 59, 254-265.

Kumar, A., Schwarz, L. B., \& Ward, J. E. (1995). Risk-pooling along a fixed delivery route using a dynamic inventory-allocation policy. Management Science, 41, 344-362.

Lee, H. L., Padmanabhan, V., \& Whang, S. J. (1997). Information distortion in a supply chain: The bullwhip effect. Management Science, 43(4), 546-558.

Schwarz, L. B. (1989). A model for assessing the value of warehouse riskpooling: Risk-pooling over outside-supplier leadtimes. Management Science, 35, 828-842.

Topkis, D. M. (1968). Optimal ordering and rationing policies in a nonstationary dynamic inventory model with $n$-demand classes. Management Science, 15(3), 160-176.

Zhong, K., \& Wu, D. (2013). Pien Tze Huang gets into resource trouble, "two wings" strategy is still being formulated. First Financial Daily, February 26, C01.

\section{Appendix}

Proof of Lemma 1: Note that $G_{12}\left(m_{1}, m_{2}\right)$ in Equation (12) can be written as follows:

$$
\begin{aligned}
G_{12}^{*}= & \max _{m_{1} \in\left(K-m_{2}, K\right]} G_{12}\left(m_{1}, m_{2}\right) \\
= & \left\{\begin{array}{l}
\pi_{1}^{*}=\max _{m_{1} \in\left(K-m_{2}, K\right]} \pi_{1}, \quad \text { if } m_{2} \leq(1-\alpha) K, \\
\pi_{23}^{*}=\max \left\{\pi_{2}^{*}=\max _{m_{1} \in\left(K-m_{2}, \alpha K\right]} \pi_{2} ; \quad \pi_{3}^{*}=\max _{m_{1} \in(\alpha K, K]} \pi_{3}\right\}, \\
\text { if } m_{2} \in((1-\alpha) K, K],
\end{array}\right.
\end{aligned}
$$


where

$$
\begin{aligned}
\pi_{1} & =(M-K-w)\left(K-m_{2}\right), \\
\pi_{2} & =(M-K-w) m_{1}, \\
\pi_{3} & =(M-K-w) \alpha K .
\end{aligned}
$$

Namely, we divide $G_{12}\left(m_{1}, m_{2}\right)$ into two subscenarios based on retailer 2's order quantity: subscenario $1: m_{2} \leq(1-\alpha) K$, and subscenario $2: m_{2} \in((1-\alpha) K, K]$.

Immediately, we can gain the optimal value $\pi_{1}^{*}=(M-K-w)\left(K-m_{2}\right)$ and optimal solution $m_{12-1}^{*} \in\left(K-m_{2}, K\right]$ with the subscenario 1: $m_{2} \leq(1-$ $\alpha) K$.

Under the subscenario 2: $m_{2} \in((1-\alpha) K, K]$, we have

$$
\begin{gathered}
m_{12-2}^{*}=\arg \max _{m_{1} \in\left(K-m_{2}, \alpha K\right]} \pi_{2}= \begin{cases}\left(K-m_{2}\right)^{+}, & \text {if } M-w \leq K, \\
\alpha K, & \text { if } M-w>K,\end{cases} \\
\pi_{2}^{*}= \begin{cases}(M-K-w)\left(K-m_{2}\right)^{+}, & \text {if } M-w \leq K, \\
(M-K-w) \alpha K, & \text { if } M-w>K,\end{cases}
\end{gathered}
$$

and

$$
\begin{aligned}
m_{12-3}^{*} & =\arg \max _{m_{1} \in\left(K-m_{2}, \alpha K\right]} \pi_{3} \in[\alpha K, K], \\
\pi_{3}^{*} & =\max _{m_{1} \in(\alpha K, K]} \pi_{3}=(M-K-w) \alpha K .
\end{aligned}
$$

Comparing $\pi_{2}^{*}$ in Equation (A.2) with $\pi_{3}^{*}$ in Equation (A.3), we can directly gain the following result with condition $m_{2} \in((1-\alpha) K, K]$ holding:

$$
\begin{aligned}
& m_{23}^{*}=\arg \max _{m_{1}}\left\{\pi_{2}^{*} ; \pi_{3}^{*}\right\}= \begin{cases}\left(K-m_{2}\right)^{+} & \text {if } M-w \leq K, \\
\in[\alpha K, K] & \text { if } M-w>K .\end{cases} \\
& \pi_{23}^{*}=\max \left\{\pi_{2}^{*} ; \pi_{3}^{*}\right\}= \begin{cases}(M-K-w)\left(K-m_{2}\right)^{+}, & \text {if } M-w \leq K, \\
(M-K-w) \alpha K, & \text { if } M-w>K .\end{cases}
\end{aligned}
$$

Consequently, by the analysis of subscenario 1 and subscenario 2 , we prove the lemma.

Proof of Theorem 1: To prove the theorem, we first introduce Lemma 5.

Lemma 5: Under condition $M-w \in[K,(1+\alpha) K]$, if $m_{2} \leq \hat{\alpha}$ or $m_{2} \geq M-$ $w+2 \sqrt{(M-w-K) \alpha K}$, then $\frac{\left(M-w-m_{2}\right)^{2}}{4} \geq(M-w-K) \alpha K$. Furthermore, we have

a) $M-w+2 \sqrt{(M-w-K) \alpha K}>2 K-(M-w)$;

b) $\hat{\alpha}>(1-\alpha) K$

c) $\hat{\alpha}<2 K-(M-w)$.

Proof: Under condition $M-w \in[K,(1+\alpha) K]$, inequality $\frac{\left(M-w-m_{2}\right)^{2}}{4} \geq(M-$ $w-K) \alpha K$ is equivalent to

$$
m_{2}^{2}-2(M-w) m_{2}+(M-w)^{2}-4(M-w-K) \alpha K \geq 0
$$


and is also equivalent to

$m_{2} \geq M-w+2 \sqrt{(M-w-K) \alpha K}$ or $m_{2} \leq M-w-2 \sqrt{(M-w-K) \alpha K}$.

Note that $M-w+2 \sqrt{(M-w-K) \alpha K}>2 K-(M-w) \quad$ is equivalent to $2(M-w-K)+2 \sqrt{(M-w-K) \alpha K}>0$, and that $M-w-$ $2 \sqrt{(M-w-K) \alpha K}>(1-\alpha) K$ is equivalent to $M-w-(1-\alpha) K>$ $2 \sqrt{(M-w-K) \alpha K}$. Further, we have that $M-w-2 \sqrt{(M-w-K) \alpha K}<$ $2 K-(M-w)$. Thus, the lemma holds.

Now we prove the theorem. By Equation (10), we compare $G_{11}^{*}$ and $G_{12}^{*}$ according the range of $w$, which can be divided into two cases:

Case 1: $w \geq M-K$. In the case, we have

$$
\begin{aligned}
& G_{11}^{*}= \begin{cases}0, \quad m_{11}^{*}=0 & \text { if } m_{2} \in(M-w, K], \\
\frac{\left(M-w-m_{2}\right)^{2}}{4}, \quad m_{11}^{*}=\frac{M-w-m_{2}}{2} & \text { if } m_{2} \in[0, M-w],\end{cases} \\
& G_{12}^{*}=\left\{\begin{array}{l}
(M-K-w)\left(K-m_{2}\right), \quad m_{12}^{*} \in\left[K-m_{2}, K\right] \text { if } m_{2} \in[0,(1-\alpha) K], \\
(M-K-w)\left(K-m_{2}\right)^{+}, \quad m_{12}^{*}=\left(K-m_{2}\right)^{+} \text {if } m_{2} \in((1-\alpha) K, K] .
\end{array}\right.
\end{aligned}
$$

Case 1 can be divided into the following two subcases to analyze.

Case 1.a. $w \in(M-(1-\alpha) K, M]$.

In this subcase, $0 \leq M-w<(1-\alpha) K<K$. Thus, we have:

if $0 \leq m_{2}<M-w$, then $G_{11}^{*}=\frac{\left(M-w-m_{2}\right)^{2}}{4}>G_{12}^{*}=(M-K-w)(K-$ $\left.m_{2}\right)$. Hence, $\Pi_{1}\left(w, m_{2}\right)=\frac{\left(M-w-m_{2}\right)^{2}}{4}$ and $m_{1}\left(m_{2}\right)=m_{11}^{*}=\frac{M-w-m_{2}}{2}$;

if $m_{2} \in[M-w, K]$, then $G_{11}^{*}=0$ and $G_{12}^{*}$ is negative. Hence, $\Pi_{1}\left(w, m_{2}\right)=$ 0 and $m_{1}\left(m_{2}\right)=m_{11}^{*}=0$.

Case 1.a is illustrated as follows:

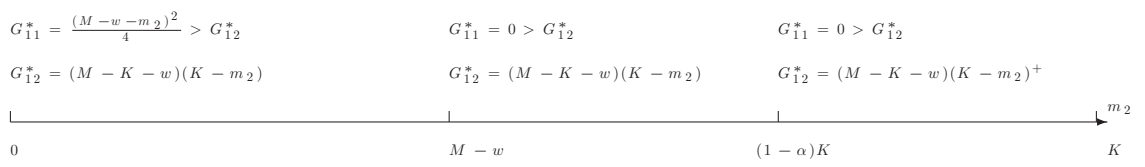

Case 1.b. $w \in[M-K, M-(1-\alpha) K]$.

In this subcase, we have $0<(1-\alpha) K<M-w<K$. Thus, we have:

if $m_{2} \in(0,(1-\alpha) K]$, then $G_{11}^{*}=\frac{\left(M-w-m_{2}\right)^{2}}{4}$ and $G_{12}^{*}=(M-K-$ $w)\left(K-m_{2}\right)$. We have that $G_{11}^{*}>G_{12}^{*}$. Hence, $\Pi_{1}\left(w, m_{2}\right)=\frac{\left(M-w-m_{2}\right)^{2}}{4}$ and $m_{1}\left(m_{2}\right)=m_{11}^{*}=\frac{M-w-m_{2}}{2}$;

if $m_{2} \in((1-\alpha) K, M-w]$, then $G_{11}^{*}=\frac{\left(M-w-m_{2}\right)^{2}}{4}$, and $G_{12}^{*}=(M-K-$ $w)\left(K-m_{2}\right)^{+}$is negative. Thus, we have that $G_{11}^{*}>G_{12}^{*}$. Hence, $\Pi_{1}\left(w, m_{2}\right)=$ $\frac{\left(M-w-m_{2}\right)^{2}}{4}$ and $m_{1}\left(m_{2}\right)=m_{11}^{*}=\frac{M-w-m_{2}}{2}$;

if $m_{2} \in(M-w, K]$, then $G_{11}^{*}=0$ and $G_{12}^{*}=(M-K-w)\left(K-m_{2}\right)^{+}$is negative. Thus, we have that $G_{11}^{*}>G_{12}^{*}$. Hence, $\Pi_{1}\left(w, m_{2}\right)=0$ and $m_{1}\left(m_{2}\right)=$ $m_{11}^{*}=0$. 
Case 1.b is illustrated as follows:

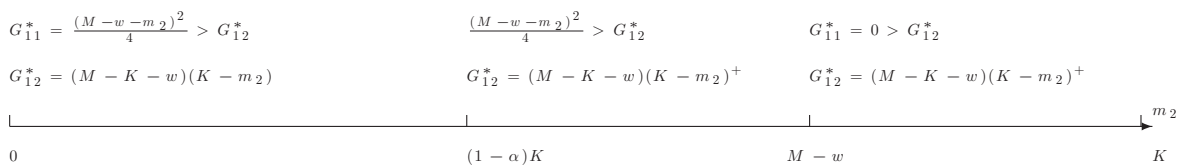

Combining cases 1.a and 1.b, we have (i).

Case 2: $w<M-K$. In this subcase, we have

$$
\begin{aligned}
& G_{11}^{*}= \begin{cases}\frac{\left(M-w-m_{2}\right)^{2}}{4}, \quad m_{11}^{*}=\frac{M-w-m_{2}}{2} & \text { if } m_{2} \leq 2 K-(M-w), \\
(M-K-w)\left(K-m_{2}\right), \quad m_{12}^{*}=K-m_{2} \text { if } m_{2} \in(2 K-(M-w), K],\end{cases} \\
& G_{12}^{*}= \begin{cases}(M-K-w)\left(K-m_{2}\right), \quad m_{1}^{*} \in\left[K-m_{2}, K\right] & \text { if } m_{2} \in[0,(1-\alpha) K], \\
(M-K-w) \alpha K, \quad m_{1}^{*} \in[\alpha K, K] & \text { if } m_{2} \in((1-\alpha) K, K] .\end{cases}
\end{aligned}
$$

The case can be divided into the following three subcases to analyze:

Case 2.a. $w \in(M-(1+\alpha) K, M-K)$.

In this subcase, we know that $0<(1-\alpha) K<\hat{\alpha}<2 K-(M-w)<K$. By Lemma 5, we consider the following conditions:

if $m_{2} \in[0,(1-\alpha) K)$, then $G_{11}^{*}=\frac{\left(M-w-m_{2}\right)^{2}}{4}$ and $G_{12}^{*}=(M-K-$ $w)\left(K-m_{2}\right)$, which implies $G_{11}^{*}>G_{12}^{*}$. Therefore, we have $\Pi_{1}\left(w, m_{2}\right)=$ $\frac{\left(M-w-m_{2}\right)^{2}}{4}$ and $m_{1}\left(m_{2}\right)=\frac{M-w-m_{2}}{2}$;

if $m_{2} \in[(1-\alpha) K, \hat{\alpha}]$, then $G_{11}^{*}=\frac{\left(M-w-m_{2}\right)^{2}}{4}$ and $G_{12}^{*}=(M-K-w) \alpha K$, which implies $G_{11}^{*}>G_{12}^{*}$ by Lemma 5 . Therefore, we have $\Pi_{1}\left(w, m_{2}\right)=$ $\frac{\left(M-w-m_{2}\right)^{2}}{4}$ and $m_{1}\left(m_{2}\right)=\frac{M-w-m_{2}}{2}$;

if $m_{2} \in(\hat{\alpha}, 2 K-(M-w))$, then $G_{11}^{*}=\frac{\left(M-w-m_{2}\right)^{2}}{4}$ and $G_{12}^{*}=(M-K-$ w) $\alpha K$, which implies $G_{11}^{*} \leq G_{12}^{*}$. Therefore, we have $\Pi_{1}\left(w, m_{2}\right)=(M-K-$ $w) \alpha K$ and $m_{1}\left(m_{2}\right) \in[\alpha K, K]$

if $m_{2} \in[2 K-(M-w), K]$, then $G_{11}^{*}=(M-K-w)\left(K-m_{2}\right)$ and $G_{12}^{*}=(M-K-w) \alpha K$, which implies $G_{11}^{*}<G_{12}^{*}$. Therefore, we have $\Pi_{1}\left(w, m_{2}\right)=(M-K-w) \alpha K$ and $m_{1}\left(m_{2}\right) \in[\alpha K, K]$.

Case 2.a is illustrated as follows:

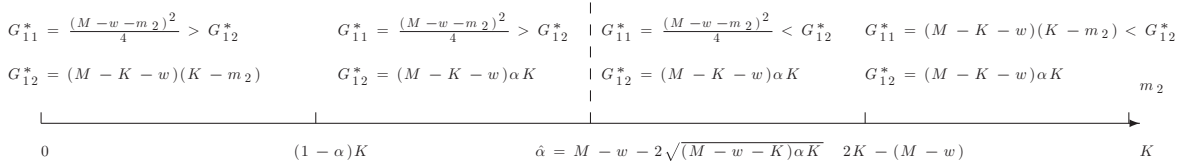

From the analysis of case 2.a, we have (ii).

Case 2.b. $w \in(M-2 K, M-(1+\alpha) K]$.

In this subcase, $0<2 K-(M-w) \leq(1-\alpha) K<K$. Thus, we have

if $m_{2} \in[0,2 K-(M-w))$, then $G_{11}^{*}=\frac{\left(M-w-m_{2}\right)^{2}}{4}$ and $G_{12}^{*}=(M-K-$ $w)\left(K-m_{2}\right)$, which implies $G_{11}^{*}>G_{12}^{*}$. Therefore, we have $\Pi_{1}\left(w, m_{2}\right)=$ $\frac{\left(M-w-m_{2}\right)^{2}}{4}$ and $m_{1}\left(m_{2}\right)=\frac{M-w-m_{2}}{2}$;

if $m_{2} \in[2 K-(M-w),(1-\alpha) K)$, then $G_{11}^{*}=(M-K-w)\left(K-m_{2}\right)$ and $G_{12}^{*}=(M-K-w)\left(K-m_{2}\right)$, which implies $G_{11}^{*}=G_{12}^{*}$. Therefore, we have $\Pi_{1}\left(w, m_{2}\right)=(M-K-w)\left(K-m_{2}\right)$ and $m_{1}\left(m_{2}\right) \in\left[K-m_{2}, K\right]$; 
if $m_{2} \in[(1-\alpha) K, K]$, then $G_{11}^{*}=(M-K-w)\left(K-m_{2}\right)$ and $G_{12}^{*}=$ $(M-K-w) \alpha K$, which implies $G_{11}^{*}<G_{12}^{*}$. Therefore, we have $\Pi_{1}\left(w, m_{2}\right)=$ $(M-K-w) \alpha K$ and $m_{1}\left(m_{2}\right) \in[\alpha K, K]$.

Case 2.b is illustrated as follows:

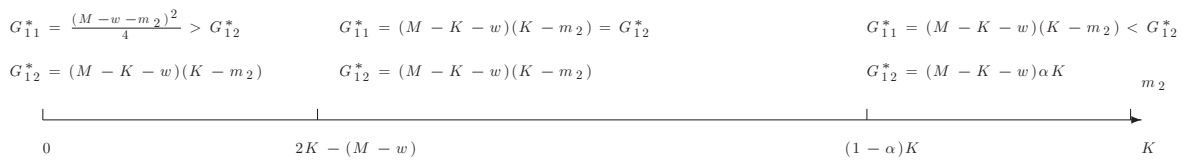

From the analysis of case 2.b, we have (iii).

Case 2.c. $w \leq M-2 K$.

In this subcase, $0<(1-\alpha) K<K$, then we have

if $m_{2} \in[0,(1-\alpha) K)$, then we can obtain that $G_{11}^{*}=(M-K-w)(K-$ $\left.m_{2}\right)$ and $G_{12}^{*}=(M-K-w)\left(K-m_{2}\right)$, which implies that $G_{11}^{*}=G_{12}^{*}$. Therefore, we have $\Pi_{1}\left(w, m_{2}\right)=(M-K-w)\left(K-m_{2}\right)$ and $m_{1}\left(m_{2}\right) \in\left[K-m_{2}, K\right]$;

if $m_{2} \in[(1-\alpha) K, K]$, then we can obtain that $G_{11}^{*}=(M-K-w)(K-$ $\left.m_{2}\right)$ and $G_{12}^{*}=(M-K-w) \alpha K$, which implies that $G_{11}^{*} \leq G_{12}^{*}$. Therefore, we have $\Pi_{1}\left(w, m_{2}\right)=(M-K-w) \alpha K$ and $m_{1}\left(m_{2}\right) \in[\alpha K, K]$.

Case 2.c is illustrated as follows:

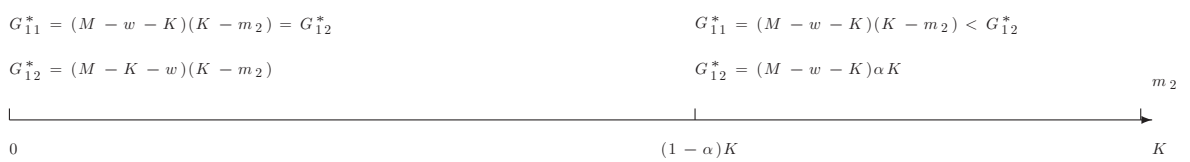

From the analysis if case 2.c, we have (iv).

Proof of Lemma 2: Note that $G_{22}\left(m_{1}, m_{2}\right)$ can be written as follows:

$$
\begin{aligned}
G_{22}^{*}= & \max _{m_{2} \in\left(K-m_{1}, K\right]} G_{22}\left(m_{1}, m_{2}\right) \\
= & \left\{\begin{array}{c}
\hat{\pi}_{1}^{*}=\max _{m_{2} \in\left(K-m_{1}, K\right]} \hat{\pi}_{1}, \\
\hat{\pi}_{23}^{*}=\max \left\{\hat{\pi}_{2}^{*}=\max _{m_{2} \in\left(K-m_{1},(1-\alpha) K\right]} \hat{\pi}_{2} ; \hat{\pi}_{3}^{*}\right. \\
\left.=\max _{m_{2} \in((1-\alpha) K, K]} \hat{\pi}_{3}\right\},
\end{array} \quad \text { if } m_{1} \leq \alpha K,\right.
\end{aligned}
$$

where

$$
\begin{aligned}
& \hat{\pi}_{1}=(M-K-w)\left(K-m_{1}\right), \\
& \hat{\pi}_{2}=(M-K-w) m_{2}, \\
& \hat{\pi}_{3}=(M-K-w)(1-\alpha) K .
\end{aligned}
$$

It is easy to obtain that

$$
\begin{aligned}
m_{21}^{*} & =\arg \max _{m_{2} \in\left(K-m_{1}, K\right]} \hat{\pi}_{1} \in\left(K-m_{1}, K\right], \\
\hat{\pi}_{1}^{*} & =(M-K-w)\left(K-m_{1}\right) .
\end{aligned}
$$

With condition $m_{1} \in(\alpha K, K]$, we have

$$
\begin{aligned}
\hat{\pi}_{2}^{*} & =\max _{m_{2} \in\left(K-m_{1},(1-\alpha) K\right]} \hat{\pi}_{2} \\
& =\left\{\begin{array}{lll}
(M-K-w)\left(K-m_{1}\right)^{+}, & \text {if } & M-w \leq K, \\
(M-K-w)(1-\alpha) K, & \text { if } & M-w>K,
\end{array}\right.
\end{aligned}
$$




$$
\hat{\pi}_{3}^{*}=\max _{m_{2} \in((1-\alpha) K, K]} \hat{\pi}_{3}=(M-K-w)(1-\alpha) K, \quad m_{2}^{*} \in((1-\alpha) K, K] .
$$

Comparing $\pi_{2}^{*}$ with $\pi_{3}^{*}$, we can directly gain the following result with condition $m_{1} \in(\alpha K, K]$ holding:

$$
\begin{aligned}
& m_{23}^{*}=\arg \max _{m_{2}}\left\{\hat{\pi}_{2}^{*} ; \hat{\pi}_{3}^{*}\right\} \\
& = \begin{cases}\left(K-m_{1}\right)^{+} & \text {if } M-w \leq K, \\
\in[(1-\alpha) K, K] & \text { if } M-w>K,\end{cases} \\
& \hat{\pi}_{23}^{*}=\max \left\{\hat{\pi}_{2}^{*} ; \hat{\pi}_{3}^{*}\right\} \\
& = \begin{cases}(M-K-w)\left(K-m_{1}\right)^{+}, & \text {if } M-w \leq K, \\
(M-K-w)(1-\alpha) K, & \text { if } M-w>K .\end{cases}
\end{aligned}
$$

Hence, the lemma holds.

Proof of Theorem 2: First, we would like to introduce the following result: inequality $\frac{\left(M-w-m_{1}\right)^{2}}{4}>(M-K-w)(1-\alpha) K$ is equivalent to $m_{1}<\beta$ or $m_{1}>$ $M-w+2 \sqrt{(M-K-w)(1-\alpha) K}$. Note that inequality $\frac{\left(M-w-m_{1}\right)^{2}}{4}>(M-$ $K-w)(1-\alpha) K$ is equivalent to $M-w-m_{1}>2 \sqrt{(M-K-w)(1-\alpha) K}$ or $M-w-m_{1}<-2 \sqrt{(M-K-w)(1-\alpha) K}$, and thus the result holds. This result will be implicitly used in the following proof.

With Equation (13) and Lemma 2, we can divide the problem into the following two cases to compare $G_{21}^{*}$ and $G_{22}^{*}$ according the range of $w$

Case 1: $w \geq M-K$. In the case, we have

$$
\begin{aligned}
G_{21}^{*} & = \begin{cases}0, \quad m_{21}^{*}=0 & \text { if } \quad m_{1} \in(M-w, K] . \\
\frac{\left(M-w-m_{1}\right)^{2}}{4}, \quad m_{21}^{*}=\frac{M-w-m_{1}}{2} & \text { if } \quad m_{1} \in[0, M-w],\end{cases} \\
G_{22}^{*} & =\left\{\begin{array}{lll}
(M-K-w)\left(K-m_{1}\right), & m_{22}^{*} \in\left[K-m_{1}, K\right] & \text { if } \quad m_{1} \in[0, \alpha K], \\
(M-K-w)\left(K-m_{1}\right)^{+}, & m_{22}^{*}=\left(K-m_{1}\right)^{+} & \text {if } \quad m_{1} \in(\alpha K, K] .
\end{array}\right.
\end{aligned}
$$

Case 1 can be divided into the following two subcases to analyze.

Case 1.a. $w \in(M-\alpha K, M]$.

In this subcase, $0 \leq M-w<\alpha K<K$. Thus, we have:

if $0 \leq m_{1}<M-w$, then $G_{21}^{*}=\frac{\left(M-w-m_{1}\right)^{2}}{4}>G_{22}^{*}=(M-K-w)(K-$ $\left.m_{1}\right)$. Hence, $\Pi_{2}\left(w, m_{1}\right)=\frac{\left(M-w-m_{1}\right)^{2}}{4}$ and $m_{2}\left(m_{1}\right)=m_{21}^{*}=\frac{M-w-m_{1}}{2}$;

if $m_{1} \in[M-w, K]$, then $G_{21}^{*}=0$ and $G_{22}^{*}$ is negative. Hence, $\Pi_{1}\left(w, m_{1}\right)=$ 0 and $m_{2}\left(m_{1}\right)=m_{21}^{*}=0$.

Case 1.a is illustrated as follows:

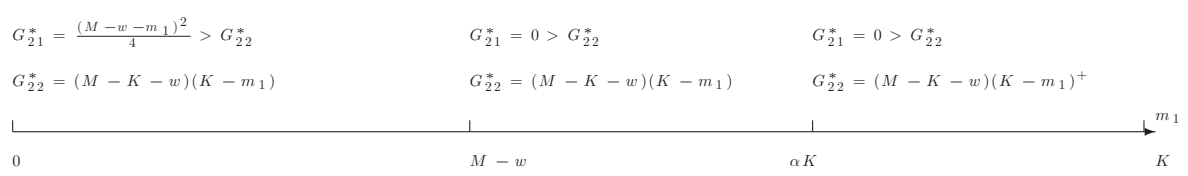

Case 1.b. $w \in[M-K, M-\alpha K]$.

In this subcase, we have $0<\alpha K<M-w<K$. Thus, we have: 
if $m_{1} \in(0, \alpha K]$, then $G_{21}^{*}=\frac{\left(M-w-m_{1}\right)^{2}}{4}$ and $G_{22}^{*}=(M-K-w)\left(K-m_{1}\right)$. We have that $G_{21}^{*}>G_{22}^{*}$. Hence, $\Pi_{2}\left(w, m_{1}\right)=\frac{\left(M-w-m_{1}\right)^{2}}{4}$ and $m_{2}\left(m_{1}\right)=m_{21}^{*}=$ $\frac{M-w-m_{1}}{2}$;

if $m_{1} \in(\alpha K, M-w]$, then $G_{21}^{*}=\frac{\left(M-w-m_{1}\right)^{2}}{4}$, and $G_{22}^{*}=(M-K-$ $w)\left(K-m_{1}\right)^{+}$is negative. Thus, we have that $G_{21}^{*}>G_{22}^{*}$. Hence, $\Pi_{2}\left(w, m_{1}\right)=$ $\frac{\left(M-w-m_{1}\right)^{2}}{4}$ and $m_{2}\left(m_{1}\right)=m_{21}^{*}=\frac{M-w-m_{1}}{2}$;

if $m_{1} \in(M-w, K]$, then $G_{21}^{*}=0$ and $G_{22}^{*}=(M-K-w)\left(K-m_{1}\right)^{+}$is negative. Thus, we have that $G_{21}^{*}>G_{22}^{*}$. Hence, $\Pi_{2}\left(w, m_{1}\right)=0$ and $m_{2}\left(m_{1}\right)=$ $m_{21}^{*}=0$.

Case 1.b is illustrated as follows:

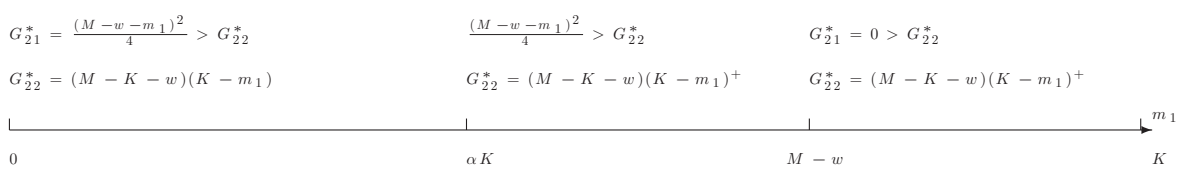

Combining cases 1.a and 1.b, we have (i).

Case 2: $w<M-K$. In this subcase, we have

$$
\begin{aligned}
& G_{21}^{*}= \begin{cases}\frac{\left(M-w-m_{1}\right)^{2}}{4}, \quad m_{2}^{*}=\frac{M-w-m_{1}}{2} & \text { if } m_{1} \leq 2 K-(M-w), \\
(M-K-w)\left(K-m_{1}\right), \quad m_{2}^{*}=K-m_{1} & \text { if } m_{1} \in(2 K-(M-w), K],\end{cases} \\
& G_{22}^{*}=\left\{\begin{array}{lll}
(M-K-w)\left(K-m_{1}\right), & m_{2}^{*} \in\left(K-m_{1}, K\right] & \text { if } \quad m_{1} \in[0, \alpha K], \\
(M-K-w)(1-\alpha) K, & m_{2}^{*} \in[(1-\alpha) K, K] & \text { if } \quad m_{1} \in(\alpha K, K] .
\end{array}\right.
\end{aligned}
$$

The case can be divided into the following three subcases to analyze:

Case 2.a. $M-w \in(M-(2-\alpha) K, M-K)$.

In this subcase, we know that $0<\alpha K<\beta<2 K-(M-w)<K$. By Lemma 5, we consider the following conditions:

if $m_{1} \in[0, \alpha K)$, then $G_{21}^{*}=\frac{\left(M-w-m_{1}\right)^{2}}{4}$ and $G_{22}^{*}=(M-K-w)\left(K-m_{1}\right)$, which implies $G_{21}^{*}>G_{22}^{*}$. Therefore, we have $\Pi_{2}\left(w, m_{1}\right)=\frac{\left(M-w-m_{1}\right)^{2}}{4}$ and $m_{2}\left(m_{1}\right)=\frac{M-w-m_{1}}{2}$;

if $m_{1} \in[\alpha K, \beta]$, then $G_{21}^{*}=\frac{\left(M-w-m_{1}\right)^{2}}{4}$ and $G_{22}^{*}=(M-K-w)(1-\alpha) K$, which implies $G_{21}^{*} \geq G_{22}^{*}$ by Lemma 5 . Therefore, we have $\Pi_{2}\left(w, m_{1}\right)=$ $\frac{\left(M-w-m_{1}\right)^{2}}{4}$ and $m_{2}\left(m_{1}\right)=\frac{M-w-m_{1}}{2}$;

if $m_{1} \in(\beta, 2 K-(M-w))$, then $G_{21}^{*}=\frac{\left(M-w-m_{1}\right)^{2}}{4}$ and $G_{22}^{*}=(M-K-$ $w)(1-\alpha) K$, which implies $G_{21}^{*}<G_{22}^{*}$. Therefore, we have $\Pi_{2}\left(w, m_{1}\right)=(M-$ $K-w)(1-\alpha) K$ and $m_{2}\left(m_{1}\right) \in[(1-\alpha) K, K]$;

if $m_{1} \in[2 K-(M-w), K]$, then $G_{21}^{*}=(M-K-w)\left(K-m_{1}\right)$ and $G_{22}^{*}=(M-K-w)(1-\alpha) K$, which implies $G_{21}^{*}<G_{22}^{*}$. Therefore, we have $\Pi_{2}\left(w, m_{1}\right)=(M-K-w)(1-\alpha) K$ and $m_{2}\left(m_{1}\right) \in[(1-\alpha) K, K]$.

Case 2.a is illustrated as follows:

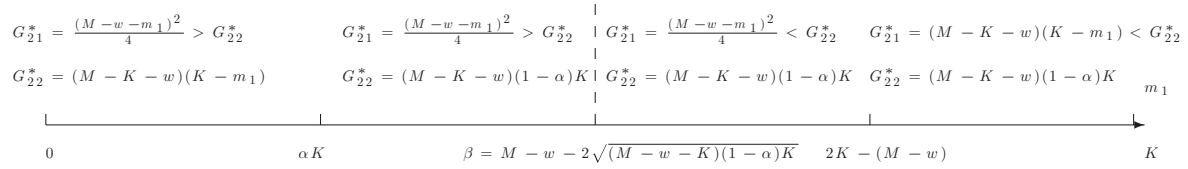

From the analysis of case 2.a, we have (ii). 
Case 2.b. $w \in(M-2 K, M-(2-\alpha) K]$.

In this subcase, $0<2 K-(M-w) \leq(2-\alpha) K<K$. Thus, we have

if $m_{1} \in[0,2 K-(M-w))$, then $G_{21}^{*}=\frac{\left(M-w-m_{1}\right)^{2}}{4}$ and $G_{22}^{*}=(M-K-$ $w)\left(K-m_{1}\right)$, which implies $G_{21}^{*}>G_{22}^{*}$. Therefore, we have $\Pi_{2}\left(w, m_{1}\right)=$ $\frac{\left(M-w-m_{1}\right)^{2}}{4}$ and $m_{2}\left(m_{1}\right)=\frac{M-w-m_{1}}{2}$;

if $m_{1} \in[2 K-(M-w), \alpha K)$, then $G_{21}^{*}=(M-K-w)\left(K-m_{1}\right)$ and $G_{22}^{*}=(M-K-w)\left(K-m_{1}\right)$, which implies $G_{21}^{*}=G_{22}^{*}$. Therefore, we have $\Pi_{2}\left(w, m_{1}\right)=(M-K-w)\left(K-m_{1}\right)$ and $m_{2}\left(m_{1}\right) \in\left[K-m_{1}, K\right]$;

if $m_{1} \in[\alpha K, K]$, then $G_{21}^{*}=(M-K-w)\left(K-m_{1}\right)$ and $G_{22}^{*}=(M-$ $K-w)(1-\alpha) K$, which implies $G_{21}^{*}<G_{22}^{*}$. Therefore, we have $\Pi_{2}\left(w, m_{1}\right)=$ $(M-K-w)(1-\alpha) K$ and $m_{2}\left(m_{1}\right) \in[(1-\alpha) K, K]$.

Case 2.b is illustrated as follows:

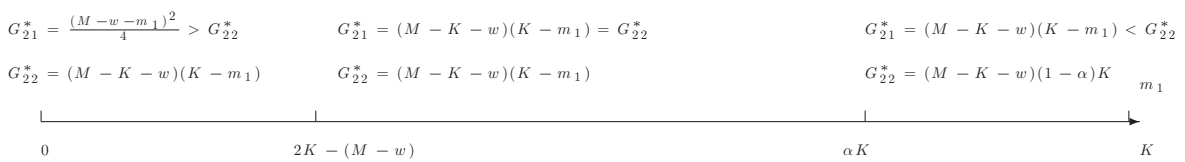

From the analysis of case 2.b, we have (iii).

Case 2.c. $w \leq M-2 K$.

In this subcase, $0<\alpha K<K$, then we have

if $m_{1} \in[0, \alpha K)$, then we can obtain that $G_{21}^{*}=(M-K-w)\left(K-m_{1}\right)$ and $G_{22}^{*}=(M-K-w)\left(K-m_{1}\right)$, which implies that $G_{21}^{*}=G_{22}^{*}$. Therefore, we have $\Pi_{2}\left(w, m_{1}\right)=(M-K-w)\left(K-m_{1}\right)$ and $m_{2}\left(m_{1}\right) \in\left[K-m_{1}, K\right]$;

if $m_{1} \in[\alpha K, K]$, then we can obtain that $G_{21}^{*}=(M-K-w)\left(K-m_{1}\right)$ and $G_{22}^{*}=(M-K-w)(1-\alpha) K$, which implies that $G_{21}^{*} \leq G_{22}^{*}$. Therefore, we have $\Pi_{2}\left(w, m_{1}\right)=(M-K-w)(1-\alpha) K$ and $m_{2}\left(m_{1}\right) \in[(1-\alpha) K, K]$.

Case 2.c is illustrated as follows:

$\begin{array}{lll}G_{21}^{*}=(M-w-K)\left(K-m_{1}\right)=G_{22}^{*} & G_{21}^{*}=(M-w-K)\left(K-m_{1}\right)<G_{22}^{*} & \\ G_{22}^{*}=(M-K-w)\left(K-m_{1}\right) & G_{22}^{*}=(M-w-K)(1-\alpha) K & m_{1} \\ \underbrace{}_{0} & 1 & \alpha K\end{array}$

From the analysis if case 2.c, we have (iv).

Proof of Theorem 3: Case 1: $w \in[0, M-2 K)$ In this case, we have:

$$
\begin{gathered}
m_{1}\left(m_{2}\right) \in \begin{cases}{\left[K-m_{2}, K\right],} & \text { if } m_{2} \in[0,(1-\alpha) K), \\
{[\alpha K, K],} & \text { if } m_{2} \in[(1-\alpha) K, K],\end{cases} \\
m_{2}\left(m_{1}\right) \in \begin{cases}{\left[K-m_{1}, K\right],} & \text { if } m_{1} \in[0, \alpha K), \\
{[(1-\alpha) K, K],} & \text { if } m_{1} \in[\alpha K, K] .\end{cases}
\end{gathered}
$$

In this case, we can obtain the Nash equilibria $m_{1}^{*} \times m_{2}^{*} \in[\alpha K, K] \times[(1-$ $\alpha) K, K]$.

Case 2: $w \in[M-2 K, M-(1+\alpha) K)$. In this case, we have:

$$
m_{1}\left(m_{2}\right) \begin{cases}=\frac{M-w-m_{2}}{2}, & \text { if } m_{2}<2 K-(M-w), \\ \in\left[K-m_{2}, K\right], & \text { if } m_{2} \in[2 K-(M-w),(1-\alpha) K), \\ \in[\alpha K, K], & \text { if } m_{2} \in[(1-\alpha) K, K]\end{cases}
$$




$$
m_{2}\left(m_{1}\right) \begin{cases}=\frac{M-w-m_{1}}{2}, & \text { if } m_{1}<2 K-(M-w), \\ \in\left[K-m_{1}, K\right], & \text { if } m_{1} \in[2 K-(M-w), \alpha K), \\ \in[(1-\alpha) K, K], & \text { if } m_{1} \in[\alpha K, K] .\end{cases}
$$

In this case, we can prove that $2 K-(M-w)<\frac{M-w}{3}$ and obtain the Nash equilibria $m_{1}^{*} \times m_{2}^{*} \in[\alpha K, K] \times[(1-\alpha) K, K]$.

Case 3: $w \in[M-(1+\alpha) K, M-(2-\alpha) K)$. In this case, we have:

$$
\begin{gathered}
m_{1}\left(m_{2}\right) \begin{cases}=\frac{M-w-m_{2}}{2}, & \text { if } m_{2} \leq \hat{\alpha}, \\
\in[\alpha K, K], & \text { if } m_{2} \in(\hat{\alpha}, K],\end{cases} \\
m_{2}\left(m_{1}\right) \begin{cases}=\frac{M-w-m_{1}}{2}, & \text { if } m_{1}<2 K-(M-w), \\
\in\left[K-m_{1}, K\right], & \text { if } m_{1} \in[2 K-(M-w), \alpha K), \\
\in[(1-\alpha) K, K], & \text { if } m_{1} \in[\alpha K, K] .\end{cases}
\end{gathered}
$$

Note that if $w \geq M-\frac{9 \alpha K-3 \sqrt{(9 \alpha-4) \alpha} K}{2}$, then there exist multiple Nash equilibria $\left(m_{1}^{*}, m_{2}^{*}\right)=\left(\frac{M-w}{3}, \frac{M-w}{3}\right)$ and $m_{1}^{*} \times m_{2}^{*} \in[\alpha K, K] \times[\hat{\alpha}, K]$, where in the later set of equilibria, we have that $g_{1}\left(m_{1}^{*}, m_{2}^{*}\right)=\alpha K$ and $g_{2}\left(m_{1}^{*}, m_{2}^{*}\right)=(1-\alpha) K$. Furthermore, the former equilibrium $\left(\frac{M-w}{3}, \frac{M-w}{3}\right)$ dominates any equilibrium belonging to $[\alpha K, K] \times[\hat{\alpha} K, K]$ in the sense of it gains larger profit for both retailers. If $w<M-\frac{9 \alpha K-3 \sqrt{(9 \alpha-4) \alpha} K}{2}$, then we have that in equilibrium, the two retailers' total order size is no less than $K$, and the equilibria are $m_{1}^{*} \times m_{2}^{*} \in[\alpha K, K] \times[\hat{\alpha}, K]$.

Case 4: $w \in[M-(2-\alpha) K, M-K)$. In this case, we have:

$$
\begin{gathered}
m_{1}\left(m_{2}\right) \begin{cases}=\frac{M-w-m_{2}}{2}, & \text { if } m_{2} \leq \hat{\alpha}, \\
\in[\alpha K, K], & \text { if } m_{2} \in(\hat{\alpha}, K],\end{cases} \\
m_{2}\left(m_{1}\right) \begin{cases}=\frac{M-w-m_{1}}{2}, & \text { if } m_{1} \leq \beta, \\
\in[(1-\alpha) K, K], & \text { if } m_{1} \in(\beta, K] .\end{cases}
\end{gathered}
$$

Note that if $w \geq M-\frac{9 \alpha K-3 \sqrt{(9 \alpha-4) \alpha} K}{2}$, then there exist multiple Nash equilibria $\left(m_{1}^{*}, m_{2}^{*}\right)=\left(\frac{M-w}{3}, \frac{M-w}{3}\right)$ and $m_{1}^{*} \times m_{2}^{*} \in[\beta, K] \times[\hat{\alpha}, K]$, where in the later set of equilibria, we have that $g_{1}\left(m_{1}^{*}, m_{2}^{*}\right)=\alpha K$ and $g_{2}\left(m_{1}^{*}, m_{2}^{*}\right)=(1-\alpha) K$. Furthermore, the former equilibrium $\left(\frac{M-w}{3}, \frac{M-w}{3}\right)$ dominates any equilibrium belonging to $[\beta, K] \times[\hat{\alpha}, K]$ in the sense that it gains larger profit for both retailers. If $w<M-\frac{9 \alpha K-3 \sqrt{(9 \alpha-4) \alpha} K}{2}$, then we have that in equilibrium, the two retailers' total order size is no less than $K$, and the equilibria are $m_{1}^{*} \times m_{2}^{*} \in[\beta, K] \times[\hat{\alpha}, K]$.

Case 5: $w \in[M-K, M]$. In this case, we have:

$$
\begin{aligned}
& m_{1}\left(m_{2}\right)= \begin{cases}\frac{M-w-m_{2}}{2}, & \text { if } m_{2}<M-w, \\
0, & \text { if } m_{2} \in[M-w, K],\end{cases} \\
& m_{2}\left(m_{1}\right)= \begin{cases}\frac{M-w-m_{1}}{2}, & \text { if } m_{1}<M-w, \\
0, & \text { if } m_{1} \in[M-w, K] .\end{cases}
\end{aligned}
$$

From the equations, we can gain the Nash equilibrium $\left(m_{1}^{*}, m_{2}^{*}\right)=\left(\frac{M-w}{3}, \frac{M-w}{3}\right)$. Combining cases $1-5$, the theorem holds.

Proof of Lemma 3: (i) To prove $3 K-3 \sqrt{\sqrt{(9 \alpha-4) \alpha}+1-3 \alpha}$ is decreasing in $\alpha \in\left[\frac{1}{2}, 1\right]$ is equivalent to prove that $\sqrt{\sqrt{(9 \alpha-4) \alpha}+1-3 \alpha}$ is increasing in 
$\alpha \in\left[\frac{1}{2}, 1\right]$, which is further equivalent to

$$
\sqrt{[9(\alpha+\Delta)-4](\alpha+\Delta)}+1-3(\alpha+\Delta)>\sqrt{(9 \alpha-4) \alpha}+1-3 \alpha,
$$

where $\Delta>0$. After straightforward algebra operations, we have proven (i).

Part (ii) can be proven similarly.

Proof of Theorem 4: In view of Equation (14), the supplier will choose his optimal wholesale price to maximize her profit:

$$
\max \left\{\max _{w \in\left(0, M-\frac{9 \alpha K-3 \sqrt{(9 \alpha-4) \alpha} K}{2}\right)} w K ; \max _{w \in\left[M-\frac{9 \alpha K-3 \sqrt{(9 \alpha-4) \alpha} K}{2}, M\right]} \frac{2(M-w) w}{3}\right\} .
$$

Note that

$$
\begin{gathered}
K\left(M-\frac{9 \alpha K-3 \sqrt{(9 \alpha-4) \alpha} K}{2}\right)>\left(M-\frac{9 \alpha K-3 \sqrt{(9 \alpha-4) \alpha} K}{2}\right) \\
(3 \alpha-\sqrt{(9 \alpha-4) \alpha)} K \\
\arg \max _{w}\left\{\frac{2(M-w) w}{3}\right\}=\frac{M}{2}, \\
\max _{w}\left\{\frac{2(M-w) w}{3}\right\}=\frac{M^{2}}{6} .
\end{gathered}
$$

Therefore, if $\frac{M}{2} \leq M-\frac{9 \alpha K-3 \sqrt{(9 \alpha-4) \alpha} K}{2}$, i.e., $M \geq 9 \alpha K-3 \sqrt{(9 \alpha-4) \alpha} K$, then by (A.7) and (A.8), we have $w^{*}(\alpha)=\left(M-\frac{9 \alpha K-3 \sqrt{(9 \alpha-4) \alpha} K}{2}\right)^{-}$and $\Pi_{s}^{*}(\alpha)=$ $K\left[\left(M-\frac{9 \alpha K-3 \sqrt{(9 \alpha-4) \alpha} K}{2}\right)^{-}\right]$. show that

When $\frac{M}{2}>M-\frac{9 \alpha K-3 \sqrt{(9 \alpha-4) \alpha} K}{2}$, i.e., $M<9 \alpha K-3 \sqrt{(9 \alpha-4) \alpha} K$, we can

$$
\begin{aligned}
& K\left[\left(M-\frac{9 \alpha K-3 \sqrt{(9 \alpha-4) \alpha} K}{2}\right)-\right] \leq \frac{M^{2}}{6} \\
& \quad \text { for } M \in(0,3 K-3 \sqrt{\sqrt{(9 \alpha-4) \alpha}+1-3 \alpha} K]
\end{aligned}
$$

and $K\left[\left(M-\frac{9 \alpha K-3 \sqrt{(9 \alpha-4) \alpha} K}{2}\right)-\right]>\frac{M^{2}}{6}$

for $M \in(3 K-3 \sqrt{\sqrt{(9 \alpha-4) \alpha}+1-3 \alpha} K, 9 \alpha K-3 \sqrt{(9 \alpha-4) \alpha} K)$.

Hence, we have that

if $M \in(0,3 K-3 \sqrt{\sqrt{(9 \alpha-4) \alpha}+1-3 \alpha} K]$, then by (A.8), (A.9), and (A.10), we have that $w^{*}(\alpha)=\frac{M}{2}$ and $\Pi_{s}^{*}(\alpha)=\frac{M^{2}}{6}$; 
if $M \in(3 K-3 \sqrt{\sqrt{(9 \alpha-4) \alpha}+1-3 \alpha} K, 9 \alpha K-3 \sqrt{(9 \alpha-4) \alpha} K)$, then by (A.8), (A.9), and (A.11), we have $w^{*}=M-\frac{9 \alpha K-3 \sqrt{(9 \alpha-4) \alpha} K}{2}$ and $\Pi_{s}^{*}(\alpha)=$ $K\left[\left(M-\frac{9 \alpha K-3 \sqrt{(9 \alpha-4) \alpha} K}{2}\right)^{-}\right]$.

Consequently, the theorem holds.

Proof of Theorem 5: The theorem follows Theorem 4 with straightforward algebra operations, and hence we omit the proof for preciseness.

Proof of Lemma 4: The lemma can be proven using straightforward algebra operations, and hence we omit the proof for preciseness.

Proof of Theorem 6: Part (i) follows Theorem 4 and Proposition 1.

If $M<3(2-\sqrt{2}) K$, then retailers' profits are $\Pi_{1}^{*}=M^{2} / 36, \Pi_{2}^{*}=M^{2} / 36$ and $\Pi_{1}^{*}+\Pi_{2}^{*}=M^{2} / 18$ under proportional allocation, the same as under fixed factor allocation with $\alpha=\frac{\sqrt{2}}{2}$. If $M \geq 3(2-\sqrt{2}) K$, then retailers' profits are $\Pi_{1}^{*}=\frac{3 \sqrt{2}-4}{2} K^{2}, \Pi_{2}^{*}=\frac{3 \sqrt{2}-4}{2} K^{2}$, and $\Pi_{1}^{*}+\Pi_{2}^{*}=(3 \sqrt{2}-4) K^{2}$ under proportional allocation. Under fixed factor allocation with $\alpha=\frac{\sqrt{2}}{2}$, retailers' profits are $\Pi_{1}^{*}(\alpha)=(3-2 \sqrt{2}) K^{2}>\Pi_{1}^{*}, \Pi_{2}^{*}(\alpha)=(5 \sqrt{2}-7) K^{2}<\Pi_{2}^{*}$, and $\Pi_{1}^{*}(\alpha)+$ $\Pi_{2}^{*}(\alpha)=(3 \sqrt{2}-4) K^{2}=\Pi_{1}^{*}+\Pi_{2}^{*}$. Hence, part (ii) of the theorem holds.

Proof of Theorem 7: Following Equation (15), the proof is divided into two cases.

Case 1. If $K \in\left[0, \frac{M}{3-3 \sqrt{\sqrt{(9 \alpha-4) \alpha}+1-3 \alpha}}\right)$, then the first order condition is

$$
\frac{\partial \hat{\Pi}_{s}^{*}(K)}{\partial K}=(M-c)-(9 \alpha-3 \sqrt{(9 \alpha-4) \alpha}) K=0 .
$$

which follows from $0<\frac{(M-c)}{9 \alpha-3 \sqrt{(9 \alpha-4) \alpha}}<\frac{M}{3-3 \sqrt{\sqrt{(9 \alpha-4) \alpha}+1-3 \alpha}}$. Hence,

$$
\begin{aligned}
& \arg \underset{K \in\left[0, \frac{M}{3-3 \sqrt{\sqrt{(9 \alpha-4) \alpha}+1-3 \alpha}}\right]}{ }\left\{\left(M-\frac{9 \alpha-3 \sqrt{(9 \alpha-4) \alpha}}{2} K\right) K-c K\right\} \\
& =\frac{M-c}{9 \alpha-3 \sqrt{(9 \alpha-4) \alpha}} \text {, } \\
& \max _{K \in\left[0, \frac{M}{3-3 \sqrt{\sqrt{(9 \alpha-4) \alpha}+1-3 \alpha}}\right]}\left\{\left(M-\frac{9 \alpha-3 \sqrt{(9 \alpha-4) \alpha}}{2} K\right) K-c K\right\} \\
& =\frac{(M-c)^{2}}{2(9 \alpha-3 \sqrt{(9 \alpha-4) \alpha})} \text {. }
\end{aligned}
$$

Case 2. It is clear that

$$
\begin{aligned}
& \arg \max _{K \in\left[\frac{M}{3-3 \sqrt{\sqrt{(9 \alpha-4) \alpha+1-3 \alpha}}}, \infty\right]}\left\{\frac{M^{2}}{6}-c K\right\}=\frac{M}{3-3 \sqrt{\sqrt{(9 \alpha-4) \alpha}+1-3 \alpha}}, \\
& \max _{K \in\left[\frac{M}{3-3 \sqrt{\sqrt{(9 \alpha-4) \alpha}+1-3 \alpha}}, \infty\right]}\left\{\frac{M^{2}}{6}-c K\right\}=\frac{M^{2}}{6}-\frac{c M}{3-3 \sqrt{\sqrt{(9 \alpha-4) \alpha}+1-3 \alpha}} .
\end{aligned}
$$


Note that

$$
\begin{aligned}
\frac{(M-c)^{2}}{2(9 \alpha-3 \sqrt{(9 \alpha-4) \alpha})} & -\left(\frac{M^{2}}{6}-\frac{c M}{3-3 \sqrt{\sqrt{(9 \alpha-4) \alpha}+1-3 \alpha}}\right) \\
= & {[M(\sqrt{\sqrt{(9 \alpha-4) \alpha}-3 \alpha+1})+c]^{2}>0 . }
\end{aligned}
$$

So, the optimal capacity is that $K^{*}(\alpha)=\frac{M-c}{9 \alpha-3 \sqrt{(9 \alpha-4) \alpha}}$. Using Theorem 4 and Equation (15), the optimal wholesale price and profit are $\left(\frac{M+c}{2}\right)^{-}$and $\frac{(M-c)^{2}}{2(9 \alpha-3 \sqrt{(9 \alpha-4) \alpha})}$, respectively.

Therefore, the theorem holds.

Jianbin Li is a professor at the School of Management, Huazhong University of Science and Technology. He received his PhD from Wuhan University in 2007. His research focuses on supply chain management, e-commerce, inventory control and optimization, and risk management. His work has been published in Production and Operations Management, International Journal of Production Economics, European Journal of Operational Research, Journal of Industrial and Management Optimization, International Journal of Production Research, Asia-Pacific Journal of Operational Research, Journal of System Sciences and System Engineering, Journal of Systems Science and Complexity, etc. He is a member of the Institute for Operations Research and the Management Sciences (INFORMS), and the Production and Operations Management Society (POMS).

Xueyuan Cai is a PhD candidate at the School of Management, Huazhong University of Science and Technology. She received her BS in Mathematics and Applied Mathematics from Henan University in 2013. Her research focuses on capacity allocation.

Zhixin Liu is an associate professor in decision science at the College of Business, the University of Michigan-Dearborn. He holds a BS in Computational Mathematics from Nankai University, an MS in Operations Research from Tsinghua University, and a PhD in Operations Management from the Ohio State University. His research interests include scheduling and pricing, specifically those with game issues. He has over 20 publications in academic journals. He is a member of the Institute for Operations Research and the Management Sciences (INFORMS), the Production and Operations Management Society (POMS), and the Decision Sciences Institute (DSI). He is on the Editorial Review Board of the journal Production and Operations Management. 